\title{
DEFINABLE SETS IN ORDERED STRUCTURES. I
}

\author{
ANAND PILLAY AND CHARLES STEINHORN ${ }^{1}$
}

\begin{abstract}
This paper introduces and begins the study of a well-behaved class of linearly ordered structures, the 0 -minimal structures. The definition of this class and the corresponding class of theories, the strongly 0 -minimal theories, is made in analogy with the notions from stability theory of minimal structures and strongly minimal theories. Theorems 2.1 and 2.3, respectively, provide characterizations of O-minimal ordered groups and rings. Several other simple results are collected in $\$ 3$. The primary tool in the analysis of 0 -minimal structures is a strong analogue of "forking symmetry," given by Theorem 4.2. This result states that any (parametrically) definable unary function in an 0 -minimal structure is piecewise either constant or an order-preserving or reversing bijection of intervals. The results that follow include the existence and uniqueness of prime models over sets (Theorem 5.1) and a characterization of all $\boldsymbol{\aleph}_{0}$-categorical O-minimal structures (Theorem 6.1).
\end{abstract}

1. Introduction. The class of linearly ordered structures has long been an important subject of concern to model theorists. Impressive results have been obtained in the study of models of several particular theories that extend the theory of linear order. Among those theories that have been approached successfully are Peano arithmetic, the theory of ordered abelian groups, that of real-closed fields, and that of linear order itself. Yet, very little has been done in the way of developing a general model theory for ordered structures. In this paper, we develop the model theory for a class of linearly ordered structures that we isolate by demanding that a structure in this class satisfy a condition whose effect is that the linear ordering and the algebraic part of the structure behave quite well with respect to one another.

Let $L$ be a finitary first-order language, and $\mathscr{M}$ an $L$-structure. A set of $n$-tuples $A \subseteq \mathscr{M}^{n}$ is said to be parametrically definable if there is some $L$-formula $\varphi\left(x_{1}, \ldots, x_{n}, y_{1}, \ldots, y_{k}\right)$ and $b_{1}, \ldots, b_{k} \in \mathscr{M}$ so that $A=\left\{\left(a_{1}, \ldots, a_{n}\right): \mathscr{M} \vDash\right.$ $\left.\varphi\left(a_{1}, \ldots, a_{n}, b_{1}, \ldots, b_{k}\right)\right\}$. If $A$ is definable without parameters, we simply say that $A$ is definable. Model theorists have enjoyed particular success in their efforts to determine structural properties of models of first-order theories $T$ by restricting their considerations to those $T$ for which the parametrically definable sets of $n$-tuples in models of $T$ satisfy certain conditions. We isolate the class of linearly ordered structures with which we shall be concerned in this paper by requiring that the parametrically definable subsets of an ordered structure in our class be of a particular simple form, which we now describe.

Received by the editors January 7, 1985.

1980 Mathematics Subject Classification. Primary 03C45; Secondary 06F99.

${ }^{1}$ Both authors supported by a grant from NSERC while visiting McGill University during the 1982-83 academic year. 
For the remainder of this paper, unless otherwise stated, we assume that $L$ contains a binary relation symbol < that is interpreted as a linear ordering in all structures that we consider. An open interval $I$ in such a structure $\mathscr{M}$ is a parametrically definable subset of $\mathscr{M}$ of the form $I=\{c \in M: \mathscr{M}=a<c<b\}$ for some $a, b \in \mathscr{M} \cup\{-\infty, \infty\}$ with $a<b$. We sometimes shall represent open intervals in $\mathscr{M}$ by $(a, b)^{\mathscr{K}}$. Similarly, we may define closed, half open-half closed, etc., intervals in $\mathscr{M}$. By an interval in $\mathscr{M}$ we shall mean, ambiguously, any of the above types of intervals in $\mathscr{M}$. An endpoint of an interval $I$ in $\mathscr{M}$ will generally be called a boundary point of $I$.

We now come to the crucial definition of this paper.

Definition 1.1. A linearly ordered structure $\mathscr{M}$ is said to be 0 -minimal if any parametrically definable subset of $\mathscr{M}$ is a finite union of intervals in $\mathscr{M}$. A first-order theory $T$ is said to be strongly O-minimal if every model of $T$ is O-minimal.

These definitions were directly inspired by the paper of van den Dries [3]. There he studies expansions of $(R,<)$ that, in our parlance, are $\mathcal{O}$-minimal.

The work of Baldwin and Lachlan [1] on strongly minimal theories serves as another source of stimulation for our work. Recall that a theory $T$ is strongly minimal if all parametrically definable subsets in any model of $T$ are either finite or cofinite. That is, the parametrically definable subsets of a model of a strongly minimal theory $T$ are precisely those which must be there as long as the language $L$ contains equality. Similarly, bearing in mind that a boolean combination of intervals in a structure $\mathscr{M}$ is also a union of intervals in $\mathscr{M}$, we see that a structure is O-minimal if its parametrically definable subsets are no more than those that must be there in the presence of a linear ordering. Consequently, 0 -minimal models are those ordered structures whose parametrically definable subsets are as simple as possible, and so perhaps from the class of ordered structures for which a general model theory is most likely to be found.

O-minimal structures have several very nice properties that follow almost immediately from the definitions. As a first illustration, let us call an ordered structure $\mathscr{M}$ definably complete if any parametrically definable subset of $\mathscr{M}$ that is bounded above (respectively, bounded below) in $\mathscr{M}$ has a least upper bound (resp., greatest lower bound) in $\mathscr{M}$. This definition, of course, is a definable analogue of the usual Dedekind completeness that $(R,<)$ enjoys. We then have

Proposition 1.2. Any O-minimal structure is definably complete.

We will invoke this useful property of $O$-minimal structures freely in what follows. Notice that the converse is not true, however. For example, the structure $(Q,<, P)$, where $P$ is a unary predicate interpreted as $P=\{1 / n: n<\omega\}$, is definably complete but not $\mathcal{O}$-minimal. As a second illustration of those properties of $\mathcal{O}$-minimal structures that are easy consequences of the definition, we observe that such structures with discrete order type-i.e., such that every element except the last, if it exists, has an immediate successor, and every element except the first, if it exists, has an immediate predecessor-behave particularly well. 
Proposition 1.3. An O-minimal structure with a definable element, whose order type is discrete, has definable Skolem functions.

Proof. Any interval in such a structure that is not the entire structure has a least or greatest element. Since any parametrically definable subset of such a structure is a finite union of intervals in the structure, it is now a routine matter to define Skolem functions.

Several frequently encountered algebraic objects are $\mathcal{O}$-minimal. We collect these examples below.

Proposition 1.4. Any of the following structures is O-minimal:

(i) a discrete linear order with or without endpoints in the language $L=\{<\}$,

(ii) a dense linear order with or without endpoints in the language $L=\{<\}$,

(iii) a divisible ordered abelian group in the language $L=\{+, 0,<\}$,

(iv) a real closed field in the language $L=\{+, \cdot, 0,1,<\}$.

Proof. That each such structure is 0 -minimal follows by elimination of quantifiers. The quantifier elimination for (i) and (ii) is due to Langford [7]. Robinson [14] proved quantifier elimination for the theory of divisible ordered abelian groups, and Tarski [15] established it for the theory of real closed fields.

In $\S 2$, we will see that the only ordered groups that are $\mathcal{O}$-minimal are precisely the divisible ordered abelian groups, and that the only 0 -minimal ordered rings are the real closed fields. Thus, the model-theoretic assumption of $\mathcal{O}$-minimality has real algebraic consequences. The situation for $\mathcal{O}$-minimal linear orderings is, not unexpectedly, more complicated. Nonetheless, we are able to characterize the 0 -minimal linear orderings (cf. §3).

This paper is organized into six sections, including this introduction. We will briefly survey our results.

$\$ 2$ contains the proofs of the converses to Proposition 1.4(iii) and (iv), mentioned above, viz., that the only strongly 0 -minimal theories of ordered groups and rings are, respectively, the theories of divisible ordered abelian groups and real closed fields.

$\$ 3$ consists of several results that do not require particularly heavy machinery to prove. The section contains two main results. The first, Theorem 3.4, generalizes to all strongly 0 -minimal theories the result of Erdös, Gillman and Henriksen [4] that any two real closed fields whose order types are the same uncountable saturated ordering are isomorphic. The second, Theorem 3.12, referred to above, characterizes all $\mathcal{O}$-minimal linear orderings in the language $L=\{<\}$.

In $\S 4$ we prove the fundamental technical tool needed to obtain the deeper result about 0 -minimal models. This proposition, Theorem 4.2 , asserts that any parametrically definable unary function in an 0 -minimal structure is piecewise monotone or constant and continuous. Theorem 4.2 illustrates how the presence of a linear order actually may enrich the structure that is possible in a model of a first-order theory. Let us make this more precise. It happens that the primary technical lemma needed for working with strongly minimal theories is the algebraic Exchange Lemma. 
Strongly 0 -minimal theories do satisfy this lemma-cf. Theorem 4.1 - but it is not strong enough to yield the results that will be proved in $\$ \S 5$ and 6 . In fact, Theorem 4.2 actually can be viewed as a strengthening of the Exchange Lemma, as we show in the comments following the statement of Theorem 4.2.

We prove in $\$ 5$ that for any substructure of a model of a strongly $\mathcal{O}$-minimal theory, there exists a model of the theory that is prime over the given substructure and, moreover, is unique up to isomorphism over the substructure. This is Theorem 5.1. It may be viewed as a generalization of the basic algebraic proposition that any ordered field is contained in a real closure that is unique up to isomorphism over the given ordered field. We remark that Shelah [16] proved the analogous result for $\omega$-stable theories, a class of theories that contains, but is much larger than, the strongly minimal theories. The existence of prime models over arbitrary substructures follows quite easily from Lemma 3.1, which asserts that "the isolated types are dense." The real difficulty in the proof of Theorem 5.1 lies rather in establishing the uniqueness of the prime model over the given substructure. Let us note here, however, that Theorem 5.1 seems to be quite tight. The mere existence of a model prime over a substructure can fail even for a structure that is very "close" to being O-minimal. For example, consider the structure $Q^{*}=(Q,<, P)$, where $P$ is a unary predicate such that both $P$ and $Q \backslash P$ are dense in $Q$. Although $Q^{*}$ "breaks up" into two substructures, $P$ and $Q \backslash P$, which by themselves are $\mathcal{O}$-minimal, it is not difficult to see that there is no prime model over $P$.

In $\S 6$, we completely characterize the $\boldsymbol{\aleph}_{0}$-categorical strongly $\mathcal{O}$-minimal theories and their countable models. This result, Theorem 6.1, also yields Corollary 6.2, which says that any $\boldsymbol{\aleph}_{0}$-categorical strongly $\mathcal{O}$-minimal theory is finitely axiomatizable. In contrast to what may occur with an $\boldsymbol{\aleph}_{0}$-categorical strongly minimal theory, the presence of a linear ordering actually forecloses the possibility of nontrivial structure. Specifically, the unique countable model of an $\boldsymbol{\aleph}_{0}$-categorical strongly $\mathcal{O}$-minimal theory essentially is completely determined by the definable partial functions of one variable that exist in the model. By contrast, "nontrivial" $\aleph_{0}$-categorical strongly minimal theories are well known: infinite-dimensional affine or projective space over a finite field, for example.

Most of the notation and terminology used in this paper is standard. Models will be denoted by $\mathscr{M}, \mathscr{N}, \ldots$, and their universes by $M, N, \ldots$, respectively. Elements of models will be denoted by the lowercase letters $a, b, c, \ldots$, and subsets of models are designated by the capital roman letters $A, B, C, \ldots$. Recall that $a \in \mathscr{M}$ is said to be algebraic over $A \subseteq \mathscr{M}$ if there is some formula $\varphi\left(x, y_{1}, \ldots, y_{n}\right)$ and $b_{1}, \ldots, b_{n} \in A$ so that

$$
\mathscr{M} \vDash \varphi\left(a, b_{1}, \ldots, b_{n}\right) \wedge \exists^{<\infty} x \varphi\left(x, b_{1}, \ldots, b_{n}\right) .
$$

If, in addition, $a$ is the unique element in $\mathscr{M}$ satisfying $\varphi\left(x, b_{1}, \ldots, b_{n}\right)$, then $a$ is said to be definable over $A$. For $A \subseteq \mathscr{M}$, the algebraic closure of $A$ in $\mathscr{M}$, denoted $\operatorname{cl}(A)$, is given by

$\{a \in \mathscr{M}: a$ is algebraic over $A\}$. 
Similarly, the definable closure of $A$ in $\mathscr{M}$, written $\operatorname{dcl}(A)$, consists of those elements in $\mathscr{M}$ that are definable over $A$. Notice that $\operatorname{cl}(\operatorname{cl}(A))=\operatorname{cl}(A)$ and $\operatorname{dcl}(\operatorname{dcl}(A))=$ $\operatorname{dcl}(A)$. Also, if $A, B \subseteq \mathscr{M}$, then the algebraic closure of $B$ over $A$ in $\mathscr{M}$, denoted $\operatorname{cl}_{A}(B)$, is just $\operatorname{cl}(A \cup B)$, i.e., those elements in $\mathscr{M}$ that are algebraic over $B$ in the structure $(\mathscr{M}, a)_{a \in A}$. Similarly, the definable closure of $B$ over $A$ in $\mathscr{M}, \mathrm{dcl}_{A}(B)$, is $\operatorname{dcl}(A \cup B)$. Observe that $\operatorname{cl}_{\varnothing}(A)=\operatorname{cl}(A)$ and $\operatorname{dcl}_{\varnothing}(A)=\operatorname{dcl}(A)$.

Most of the results in this paper were announced in [10].

The authors wish to thank Professor M. Makkai of McGill University for his hospitality and the stimulation he provided throughout our most pleasant stay at McGill during the academic year 1982-83, when this work was done.

2. Algebraic results. Here we prove two theorems which demonstrate that the assumption of strong $\mathbf{O}$-minimality has tight structural consequences in algebraic contexts. The work of Reineke [13] on minimal groups (in the ordinary sense), Podewski [11] on minimal rings, and Macintyre [8] on $\omega$-stable fields indicate that these theorems are to be expected.

TheOREM 2.1. Let $\mathscr{G}=(G,+, 0,<)$ be an O-minimal ordered group. Then $\mathscr{G}$ is a divisible ordered abelian group.

The crucial ingredient in the proof is the following lemma.

Lemma 2.2. Let $\mathscr{G}=(G,+, 0,<)$ be an O-minimal group. Then the only parametrically definable subgroups of $\mathscr{G}$ are $\{0\}$ and $\mathscr{G}$.

Proof. Suppose that $\mathscr{G}$ is nontrivial and $\mathscr{H}$ is a nontrivial parametrically definable subgroup of $\mathscr{G}$ that is different from $\mathscr{G}$. We shall obtain a contradiction. Let $\mathscr{H}$ be given by $\left\{h \in G: \mathscr{G}=\varphi\left(h, g_{1}, \ldots, g_{n}\right)\right\}$.

Since $\mathscr{H}$ is nontrivial, $\mathscr{H}$ is infinite, as the infinite set $\{n h: n \in \omega\}$ is a subset of $H$, for any $h \in H, h \neq 0$. By $\mathcal{O}$-minimality, $\mathscr{H}$ contains a nontrivial interval $I$. By translation, it follows that $\mathscr{H}$ contains a largest nontrivial interval $J$ about 0 , which without loss of generality, we may assume is symmetric about 0 . Since $\mathscr{H} \nsubseteq \mathscr{G}$, the $\mathcal{O}$-minimality of $\mathscr{G}$ implies $J$ must be of the form $(-h, h)$ or $[-h, h]$ for some $h \in G$. We prove that neither case can occur. First suppose, that $J$ assumes the form $[-h, h]$. If there is no $h^{\prime} \in G \backslash H$ such that $h<h^{\prime}<2 h$, then clearly $[-2 h, 2 h] \subseteq H$, contrary to the maximality of $J$. But if $h<h^{\prime}<2 h$, then $0<h^{\prime}-h<h$, whence $h^{\prime}-h \in H$. However, it then follows that $\left(h^{\prime}-h\right)+h=h^{\prime} \in H$, again contradicting the maximality of $J$. So $J$ cannot be of the form $[-h, h]$.

Now assume that $J=(-h, h)$. Since $J \neq \varnothing$, there is some $h^{\prime} \in J$ so that $h^{\prime}>0$. It then follows that $0<h-h^{\prime}<h$, and so $h-h^{\prime} \in H$. But again this implies that $\left(h-h^{\prime}\right)+h^{\prime}=h \in H$, which is impossible. The lemma thus is proved.

Proof of Theorem 2.1. Let us first establish that $\mathscr{G}$ is abelian. For $g \in G$, consider the parametrically definable subgroup $C(g)=\{h \in G: h+g=g+h\}$. It is easy to see that $C(g)=G$ for any $g \in G$. Hence, $G$ is abelian.

Similarly, to establish that $G$ is divisible, one easily shows that the parametrically definable subgroup $n G=\{n g: g \in G\}$ is all of $G$. 
THEOREM 2.3. Let $R=(R,+, \cdot, 1,<)$ be an O-minimal ordered ring. Then $R$ is a real closed field.

For the proof of Theorem 2.3, we need two lemmas. The following is obvious.

LEMMA 2.4. A parametrically definable convex substructure of an O-minimal structure also is O-minimal.

For the statement of the second lemma, we must recall a standard definition. An ordered ring $R$ is said to have the intermediate value property if for any polynomial $p(x)$ with coefficients in $R$ and any $a, b \in R$ such that $a<b$ and $p(a) \cdot p(b)<0$, there is some $c \in R$ so that $a<c<b$ and $p(c)=0$. The following lemma is standard (cf. [2, §7.4], for example).

LEMMA 2.5. An ordered field is real closed iff it has the intermediate value property.

Proof of Theorem 2.3. We first establish that $R$ is an ordered field and then show that it is real closed.

To show that $R$ is a field, it is enough to show that the set of nonzero positive elements of $R, R^{+}$, forms an abelian group under multiplication. As a first step in showing this, given $r \in R, r \neq 0$, notice that $r R=\{r \cdot s: s \in R\}$ forms a nontrivial ordered subgroup of $R$ under addition. Hence, by Lemma $2.2, r R=R$. In particular, there is some $s \in R$ so that $r \cdot s=1$. If $r>0$, the element $s$ necessarily is positive, whereupon $R^{+}$forms an ordered group under multiplication. Since $R^{+}$is definable and convex in $R$, Lemma 2.4 implies that it must be 0 -minimal. Consequently, Lemma 2.2 implies that $R^{+}$is abelian, as desired.

It remains to see that $R$ satisfies the intermediate value property. Let $a, b \in R$, $a<b$, and $p(x)$ be a polynomial with coefficients in $R$ such that $p(a) \cdot p(b)=0$. Without loss, we may assume that $p(a)>0$ and $p(b)<0$. If there were no $c \in R$, $a<c<b$, satisfying $p(c)=0$, then $(a, b)^{R}=P^{+} \cup P^{-}$, where $P^{+}$and $P^{-}$are the parametrically definable sets given by $P^{+}=\{d \in R: a<d<b \& p(d)>0\}$ and $P^{-}=\{d \in R: a<d<b \& p(d)<0\}$. Since it has been shown that $R$ is a field, $(a, b)^{R}$ must be a densely ordered set. Also, using the ordered field axioms one easily may verify that polynomials with coefficients in $R$ are continuous parametrically definable functions in $R$ under the topology given by the ordering.

Now, if $P^{+}=(a, b)$, then one may easily check that the continuity of $p(x)$ is violated at $x=b$. Likewise, it cannot be the case that $(a, b)=P^{-}$. Thus, by the O-minimality of $R$, there must be some $c \in(a, b)$ which is a boundary point between $P^{+}$and $P^{-}$. We show that $c \notin P^{+} \cup P^{-}$. Indeed, suppose that $c \in P^{+}$. But any open interval $I$ containing $c$ must intersect both $P^{+}$and $P^{-}$. Therefore, $p^{-1}(\{d \in R: p(c) / 2<d<3 p(c) / 2\})$ does not contain any open interval, contradicting the continuity of $p$. Similarly, $c \notin P^{-}$. But then $(a, b) \neq P^{+} \cup P^{-}$, contrary to hypothesis, and the theorem is proved.

We mention that Theorems 2.1 and 2.3 rely only on the hypothesis that the structure involved is 0 -minimal, which a priori is much weaker than the assumption that the theory of the structure is strongly 0 -minimal. It is well known, however, that a minimal structure need not have a strongly minimal theory, and, in fact, the 
problem of determining whether or not a minimal field is algebraically closed remains unsolved. Remarkably enough, though, if a linearly ordered structure is O-minimal, its theory must be strongly 0 -minimal. ${ }^{2}$

3. Basic theorems. In this section, some basic results about strongly 0 -minimal theories will be proved. Most of these will be used in subsequent sections.

LEMMA 3.1. Let $\mathscr{M}$ be an $O$-minimal structure, and $A \subseteq \mathscr{M}$. Then for any formula $\varphi(\bar{x}, \bar{a})$ having parameters from $A$, there is a formula $\psi\left(\bar{x}, \bar{a}^{\prime}\right)$ also with parameters from $A$ so that

$$
\mathscr{M} \vDash \forall \bar{x} \psi\left(\bar{x}, \bar{a}^{\prime}\right) \rightarrow \varphi(\bar{x}, \bar{a})
$$

and for every formula $\theta(\bar{x}, \bar{b})$ with parameters from $A$, exactly one of

$$
\mathscr{M} \vDash \forall x\left(\psi\left(\bar{x}, \bar{a}^{\prime}\right) \rightarrow \theta(\bar{x}, \bar{b})\right)
$$

or

$$
\mathscr{M} \vDash \forall x\left(\psi\left(\bar{x}, \bar{a}^{\prime}\right) \rightarrow \neg \theta(\bar{x}, \bar{b})\right)
$$

holds.

In other words, the isolated types of $\operatorname{Th}(\mathscr{M}, a)_{a \in A}$ are dense. (I.e., any formula with parameters from $A$ is implied by a complete formula with parameters from $A$.)

Proof. We proceed by induction on the number of free variables in $\bar{x}$. The induction step here is trivial. Indeed, suppose that $\bar{x}=\left\langle x_{1}, \ldots, x_{n+1}\right\rangle$. Let $\psi\left(x_{1}, \ldots, x_{n}\right)$ be complete for $\exists x_{n+1} \varphi\left(x_{1}, \ldots, x_{n+1}, \bar{a}\right)$ and $\bar{c}=\left\langle c_{1}, \ldots, c_{n}\right\rangle$ in $\mathscr{M}$ satisfy $\psi\left(x_{1}, \ldots, x_{n}\right)$. Next, let $\theta\left(\bar{c}, x_{n+1}\right)$ be a formula with parameters from $A \cup\left\{c_{1}, \ldots, c_{m}\right\}$ which is complete for $\varphi\left(c_{1}, \ldots, c_{n}, x_{n+1}, \bar{a}\right)$. One then easily sees that $\psi\left(x_{1}, \ldots, x_{n}\right) \wedge \theta\left(x_{1}, \ldots, x_{n+1}\right)$ is complete for $\varphi\left(x_{1}, \ldots, x_{n+1}, \bar{a}\right)$.

So it remains to prove the lemma in the case $n=1$. By strong $\mathcal{O}$-minimality, the set $\Phi$ that $\varphi(x, \bar{a})$ defines in $\mathscr{M}$ is a finite union of rational intervals. If any endpoint of any of these rational intervals satisfies $\varphi(x, a)$, then the definition of that endpoint, using just the parameters $\bar{a}$, yields a complete formula. Without loss of generality then, we can assume that $\Phi$ consists of finitely many open rational intervals. Let $\varphi_{0}(x, \bar{a})$ be the formula satisfied in $\mathscr{M}$ by exactly the leftmost such interval. If $\varphi_{0}(x, \bar{a})$ is not already complete, then there is some formula $\psi\left(x, \bar{a}^{\prime}\right)$ with parameters from $A$ so that

$$
\mathscr{M} \vDash \exists x\left(\varphi_{0}(\bar{x}, \bar{a}) \wedge \psi\left(\bar{x}, \bar{a}^{\prime}\right)\right) \wedge \exists x\left(\varphi_{0}(\bar{x}, \bar{a}) \wedge \neg \psi\left(\bar{x}, \bar{a}^{\prime}\right)\right) .
$$

But then a boundary point of $\psi\left(x, \bar{a}^{\prime}\right)$ must lie inside the open rational interval defined by $\varphi_{0}(x, \bar{a})$ in $\mathscr{M}$. But as this boundary point is definable via some formula $\psi^{*}\left(x, \bar{a}^{\prime}\right)$, we thus can take this formula as the desired complete formula.

We next give a useful criterion for a subset of an O-minimal model to be an elementary submodel.

\footnotetext{
${ }^{2}$ This result will appear in a forthcoming paper by J. Knight, A. Pillay and C. Steinhorn.
} 
Lemma 3.2. Let $A \subseteq \mathscr{M}$, where $\mathscr{M}$ is O-minimal. Moreover, suppose that $A=$ $\operatorname{dcl}(A)$. Then $A \prec \mathscr{M}$ if and only if for every $a, b \in A \cup\{ \pm \infty\}$, with $a<b$, whenever $\mathscr{M} \vDash \exists x(a<x<b)$ then $\mathscr{M} \vDash a<c<b$ for some $c \in A$.

Proof. The direction from left-to-right is trivial. For the other direction assume the hypothesis and also that $\mathscr{M} \vDash \exists x \varphi(x, \bar{a})$, where $\bar{a}$ are parameters from $A$. We must show that $\mathscr{M} \vDash \varphi(b, \bar{a})$ for some $b \in A$. If the set that $\varphi(x, \bar{a})$ defines in $\mathscr{M}$ contains any of its boundary points, then we are done, since these are definable using parameters from $A$ and $A=\operatorname{dcl}(A)$. Consequently, suppose that the set that $\varphi(x, \bar{a})$ defines in $\mathscr{M}$ is a union of finitely many open rational intervals. Let $a_{0}$, $a_{1} \in A, a_{0}<a_{1}$, be the boundary points of the first such interval. But then, by hypothesis, for some $b \in A, a_{0}<b<a_{1}$, and thus $\mathscr{M} \vDash \varphi(b, \bar{a})$, as required.

The following result provides a useful characterization of $\mathcal{O}$-strongly minimal theories. Recall that a cut $C$ in an ordered structure $\mathscr{M}$ is a maximal consistent set of formulas with parameters from $\mathscr{M}$ of the form $m \leqslant x$ or $x \leqslant m$, where $m \in \mathscr{M}$.

Theorem 3.3. Let $\mathscr{M}$ be a linearly ordered structure. Then $\mathscr{M}$ is O-minimal if and only if for any cut $C$ in $\mathscr{M}$, there is a unique complete one-type with parameters from $\mathscr{M}$ which extends $C$.

Proof. The direction from left-to-right follows immediately from $\mathcal{O}$-minimality. That is, any formula $\varphi(x, \bar{m})$ with parameters from $\mathscr{M}$ partitions $\mathscr{M}$ into rational intervals such that for any such interval $I$, either $\varphi(x, \bar{m})$ holds or $\varphi(x, \bar{m})$ holds for all $x \in I$. Then, since only one interval is consistent with $C$, the value of $\varphi(x, \bar{m})$ in any type extending $C$ is determined.

Now, let us prove the reverse direction. Let $\mathscr{M}$ be a linearly ordered structure and $\varphi(x, \bar{m})$ a formula with parameters from $\mathscr{M}$. We must prove that $\{a \in \mathscr{M}: \mathscr{M} \vDash$ $\varphi(a, \bar{m})\}$ is a finite union of rational intervals in $\mathscr{M}$. Let $\theta_{1}(x, y)$ be the formula $(\forall u)(x \leqslant u \leqslant y \rightarrow \varphi(u, \bar{m}))$ and $\theta_{2}(x, y)$ be the formula $(\forall u)(x \leqslant u \leqslant y \rightarrow$ $\neg \varphi(u, \bar{m}))$. Define an equivalence relation $\approx$ on $\mathscr{M}$ by

$$
x \approx y \quad \text { iff } \quad \theta_{1}(x, y) \vee \theta_{2}(x, y) .
$$

Now, consider the structure $\mathscr{M}^{*}$ for the language $\left\{<, P_{1}, P_{2}\right\}$, where $P_{1}$ and $P_{2}$ are unary predicates whose domain consists of the $\approx$-equivalence classes of $\mathscr{M}$ in which $<$ is induced by the ordering on $\mathscr{M}$, and in which $P_{1}$ (resp. $P_{2}$ ) holds just of the $\theta_{1}$ (respectively, $\theta_{2}$ ) classes in $\mathscr{M}^{*}$. Observe that if $a, b \in \mathscr{M}^{*}, a<b$ and $\mathscr{M}^{*} \vDash P_{1}(a) \wedge P_{1}(b)$ (resp. $\mathscr{M}^{*} \vDash P_{2}(a) \wedge P_{2}(b)$ ), then there is some $c \in \mathscr{M}^{*}$, $a<c<b$, so that $\mathscr{M}^{*} \vDash P_{2}(c)$ (resp. $\mathscr{M}^{*} \vDash P_{1}(c)$ ). Furthermore, notice that each element of $\mathscr{M}^{*}$ determines a convex set in $\mathscr{M}$. We will show that $\mathscr{M}^{*}$ must be finite and that each interval in $\mathscr{M}$ determined by an element in $\mathscr{M}^{*}$ must be rational.

First, suppose that some element of $\mathscr{M}^{*}$ either does not have an immediate successor or predecessor. We show this is impossible. Without loss of generality, let $a \in \mathscr{M}^{*}$ have no immediate successor. Let $I$ be the interval in $\mathscr{M}$ determined by $a$. Then it is easily seen that both $\{\varphi(x, m)\} \cup C$ and $\{\neg \varphi(x, m)\} \cup C$ are consistent, 
where $C$ is the cut given by

$$
\{m \leqslant x:(\exists y \in I) m \leqslant y\} \cup\{x \leqslant m:(\forall y \in I) y<m\},
$$

which is contrary to hypothesis.

Thus, $\mathscr{M}^{*}$ must be discretely ordered. We now establish that $\mathscr{M}^{*}$ cannot be infinite. For if not, then $\mathscr{M}^{*}$ contains either an infinite increasing or decreasing sequence. Without loss of generality, suppose the former and let $\left\langle a_{i}: i<\omega\right\rangle$ be such a sequence, where $a_{i}<a_{j}$ if and only if $i<j$. Let the sequence of convex sets $\left\langle K_{i}\right.$ : $i\langle\omega\rangle$ in $\mathscr{M}$ correspond to $\left\langle a_{i}: i<\omega\right\rangle$. We also may assume that for each $i<\omega$, $\mathscr{M}^{*} \vDash P_{1}\left(a_{2 i}\right) \wedge P_{2}\left(a_{2 i+1}\right)$. But then it is clear that $\{\varphi(x, \bar{m})\} \cup C$ and $\{\neg \varphi(x, \bar{m})\} \cup C$ are consistent, where

$$
\begin{aligned}
C= & \left\{m \leqslant x: \text { for some } i<\omega,\left(\exists y \in K_{i}\right) m \leqslant y\right\} \\
& \cup\left\{x \leqslant m: \text { for every } i<\omega,\left(\forall y \in K_{i}\right) y \leqslant m\right\} .
\end{aligned}
$$

But this is impossible.

So $\mathscr{M}^{*}$ must be finite. It then follows that the set defined by $\varphi(x, \bar{m})$ in $\mathscr{M}$ consists of finitely many convex sets, and it only remains to prove that these are intervals. For a contradiction, suppose that some such convex set $K$ has no boundary point in $\mathscr{M}$ on the left (the proof is identical if $K$ has no boundary point in $\mathscr{M}$ on the right). In this case, the convex subset $K^{\prime}$ of the set defined by $\neg \varphi(x, \bar{m})$ which is adjacent to $K$ on the left must have no boundary point on the right. But then it is easy to see that both $\{\varphi(x, \bar{m})\} \cup C$ and $\{\neg \varphi(x, \bar{m})\} \cup C$ are consistent, where

$$
C=\{x \leqslant m:(\exists y \in I) y \leqslant m\} \cup\{m<x:(\exists y \in J) m \leqslant y\} .
$$

This contradicts the hypothesis, and so the theorem is proved.

The next theorem establishes that a strongly $O$-minimal theory $T$ is categorical relative to the class of uncountable saturated orders which are models of the theory of linear order determined by $T$.

THEOREM 3.4. Let $T$ be a strongly O-minimal theory. If $\mathscr{M} \vDash T$ and $M \uparrow\{<\}$ is an uncountable model of $T \uparrow\{<\}$ which is saturated for quantifier-free formulas, then $\mathscr{M}$ is a saturated model; i.e., up to isomorphism $\mathscr{M}$ is the unique model of $T$ whose underlying order is isomorphic to $\mathscr{M} \uparrow\{<\}$.

The following corollary first was proved by Erdös, Gillman and Henriksen in [4]. Recall than an $\eta_{\alpha}$ set is a dense linear ordering without endpoints of power $\boldsymbol{\kappa}_{\alpha}$ in which any cut given by fewer than $\aleph_{\alpha}$ formulas is realized. The $\eta_{\alpha}$ sets were introduced by Hausdorff in [6].

COROLlaRy 3.5. Any two real closed fields whose underlying order types are $\eta_{\alpha}$ sets are isomorphic.

Proof of 3.5. By 1.4(iv), the theory of real closed fields is strongly 0 -minimal. The result then follows from Theorem 3.4.

Now we prove Theorem 3.4. 
Proof of 3.4. Let $\mathscr{M}$ be as given and $A \subseteq \mathscr{M}$ be of power less than $\aleph_{\alpha}$. We must show that any complete one-type $p$ with parameters from $A$ is realized in $\mathscr{M}$. Enumerate $p$ as $\left\{\varphi_{\alpha}\left(x, a_{\alpha}\right): \alpha<\kappa\right\}$, where $\kappa<|\mathscr{M}|$. We define a consistent set of formulas $=\left\{b_{\alpha} \leqslant x: \alpha<\kappa\right\} \cup\left\{x \leqslant c_{\alpha}: \alpha<\kappa\right\}$ with parameters from $\mathscr{M}$ with the property that any $d \in \mathscr{M}$ realizing $C$ also realized $p$. This suffices, because by the saturation of $\mathscr{M} \uparrow\{<\}$, there is such a $d \in \mathscr{M}$.

Let $\mathscr{N} \succ \mathscr{M}$ and $d \in \mathscr{N}$ realize $p$. For $\alpha<\kappa$, let $I_{1}^{\alpha} \cup I_{2}^{\alpha} \cup \cdots \cup I_{m_{\alpha}}^{\alpha}$ be the finite union of rational intervals equal to the set defined by $\varphi_{\alpha}\left(x, a_{\alpha}\right)$ in $\mathscr{N}$. Suppose that $d \in I_{j}^{\alpha}$, and that the endpoints of $I_{j}^{\alpha}$ are $b_{j}^{\alpha}<c_{j}^{\alpha}$. Since $T$ is strongly $\mathcal{O}$-minimal, $\mathscr{M} \prec \mathscr{N}$ and the parameters of $\varphi_{\alpha}$ are from $A \subseteq \mathscr{M}$, we have that $b_{j}^{\alpha}$, $c_{j}^{\alpha} \in \mathscr{M}$. If we set $b_{\alpha}=b_{j}^{\alpha}$ and $c_{\alpha}=c_{j}^{\alpha}$, we clearly are done.

Corollary 3.6. Let $T$ be strongly O-minimal, $A \subseteq \mathscr{M} \vDash T$, and $|A|<\kappa$. If $T \uparrow\{<\}$ has a saturated model of power $\kappa$, then, up to isomorphism over $A$, there is $a$ unique $\mathscr{N} \vDash T, A \subseteq \mathscr{N}$, such that $\mathscr{N} \uparrow\{<\}$ is saturated for quantifier-free formulas.

Proof of 3.6. Since $L^{\prime}=L \cup\left\{c_{a}: a \in A\right\}$, the language obtained by adjoining constants for the elements of $A$, has power less than $\kappa$, the result follows immediately from the proof of 3.4 .

The following result, both in statement and proof, parallels the folklore fact that the theory of any uncountable minimal model is strongly minimal. However, whereas the reader easily may construct a countable minimal model whose theory is not strongly minimal, this is not true in the case of 0 -minimality. ${ }^{3}$

Theorem 3.7. Suppose that $\mathscr{M}$ is 0 -minimal and that $\mathscr{M} \uparrow\{<\}$ is $\omega_{1}$-saturated for quantifier-free types. Then $\mathrm{Th}(\mathscr{M})$ is strongly O-minimal.

Proof. Let $\mathscr{M}$ be as in the hypothesis. By induction on $n<\omega$, we prove that for any formula $\varphi\left(x, y_{0}, \ldots, y_{n-1}\right)$, possibly with parameters from $\mathscr{M}$, there is an $m(\varphi)<\omega$ such that for any $a_{0}, \ldots, a_{n-1} \in \mathscr{M},\left\{c \in \mathscr{M}: \mathscr{M} \vDash \varphi\left(c, a_{0}, \ldots, a_{n-1}\right)\right\}$ consists of at most $m(\varphi)$ intervals.

For $n=0$, the assertion follows immediately from the 0 -minimality of $\mathscr{M}$. For the induction step, let $\varphi\left(x, y_{0}, \ldots, y_{n}\right)$ be given. Since boundary points are definable, we may suppose that for each $a_{0}, \ldots, a_{n} \in \mathscr{M},\left\{c: \varphi\left(c, a_{0}, \ldots, a_{n}\right)\right\}$ is finite. (I.e., just modify $\varphi$ to obtain $\varphi^{*}$ so that $\mathscr{M} \vDash \varphi^{*}\left(c, a_{0}, \ldots, a_{n}\right)$ if and only if $c$ is a boundary point of $\left\{c \in \mathscr{M}: \mathscr{M} \vDash \varphi\left(c, a_{0}, \ldots, a_{n}\right)\right\}$. Then, for notational simplicity, identify $\varphi$ with $\varphi^{*}$.) Thus, we wish to prove that there is an $m(\varphi)<\omega$ so that for all $a_{0}, \ldots, a_{n} \in \mathscr{M},\left|\left\{c: \mathscr{M}=\varphi\left(c, a_{0}, \ldots, a_{n}\right)\right\}\right|<m(\varphi)$.

By induction hypothesis, for each $b \in \mathscr{M}$ there is $m(\varphi, b)<\omega$ so that for all $a_{0}, \ldots, a_{n-1} \in \mathscr{M}$,

$$
\left|\left\{c \in \mathscr{M}: \mathscr{M}=\varphi\left(c, a_{0}, \ldots, a_{n-1}, b\right)\right\}\right|<m(\varphi, b) .
$$

For a contradiction, assume that $\{m(\varphi, b): b \in \mathscr{M}\}$ is unbounded. Let

$$
\theta_{p}(x) \equiv\left(\exists x_{0}\right) \cdots\left(\exists x_{p-1}\right)(\exists \geqslant p y) \varphi\left(y, x_{0}, \ldots, x_{p-1}, x\right)
$$

\footnotetext{
${ }^{3} \mathrm{Cf}$., footnote 2 at the end of $\S 2$.
} 
with parameters from $\mathscr{M}$. Clearly, for $p<q,\left\{b \in \mathscr{M}: \mathscr{M} \vDash \theta_{q}(b)\right\} \subseteq\{b \in \mathscr{M}$ : $\left.\mathscr{M} \vDash \boldsymbol{\theta}_{p}(b)\right\}$. Also, the assumption for a contradiction implies that for each $p$, $\left|\left\{b \in \mathscr{M}: \mathscr{M} \vDash \theta_{p}(b)\right\}\right| \geqslant \boldsymbol{N}_{0}$ and, hence, contains an interval. Without loss, then, there are intervals $I_{p}=\left(c_{p}, d_{p}\right)$ so that $I_{p} \subseteq\left\{b \in \mathscr{M}: \mathscr{M} \vDash \theta_{p}(b)\right\}$ and $I_{q} \subseteq I_{p}$ whenever $p<q$. Now, the hypothesis on the saturation of $\mathscr{M}$ implies that the cut $C(x)=\left\{c_{p} \leqslant x: p<\omega\right\} \cup\left\{x \leqslant d_{p}: p<\omega\right\}$ is realized in $\mathscr{M}$ by some element $b$. But then,

$$
\left\{\left|\left\{c \in \mathscr{M}: \mathscr{M} \vDash \varphi\left(c, a_{0}, \ldots, a_{n-1}, b\right)\right\}\right|: a_{0}, \ldots, a_{n-1} \in \mathscr{M}\right\}
$$

is bounded in $\omega$, which contradicts the induction hypothesis, and so establishes the theorem.

Recall from $\S 1$, that a theory $T$ is unstable if for some $n<\omega$ and some formula $\boldsymbol{\theta}\left(x_{1}, \ldots, x_{n}, y_{1}, \ldots, y_{n}\right)$ there exists a model $\mathscr{M}$ of $T$ such that $\theta$ linearly orders some infinite collection of $n$-tuples from $\mathscr{M}$. A crude measure of instability exists: namely, whether or not a theory $T$ has the independence property (cf. [9, Chapter 7] for the definition and various basic results). That is, a theory $T$ having the independence property shares fewer properties with stable theories than a theory that lacks it. We will show that all strongly $\mathcal{O}$-minimal theories do not have the independence property. Our proof requires a further definition and a well-known lemma that gives an alternative formulation of a theory having the independence property.

Let $\mathscr{M} \prec \mathscr{N}$ and $\mathscr{M} \subseteq A \subseteq \mathscr{N}$. A type $q\left(x_{1}, \ldots, x_{m}\right)$ over $A$ is said to be a coheir of a type $p\left(x_{1}, \ldots, x_{m}\right)$ over $\mathscr{M}$ if $q \supseteq p$ every formula $\varphi\left(x_{1}, \ldots, x_{m}\right)$ in $q$ is satisfied (in $\mathscr{N}$ ) by some $m$-tuple from $\mathscr{M}$. (Cf. [9, Chapter 1] for more about coheirs.) The next lemma is due to B. Poizat, and a proof can be found in [9, Chapter 7].

LEMma 3.8. A theory $T$ has the independence property if and only if for any $\kappa \geqslant \omega$, there is $\mathscr{M} \vDash T$ of power $\kappa, \mathscr{N} \succ \mathscr{M}$ and type $p(x)$ over $\mathscr{M}, \mid\{q: q$ is a type over $\mathscr{N}$ that is a coheir of $p\} \mid \geqslant 2^{2^{\kappa}}$.

We now prove

THeOREM 3.9. Let $T$ be strongly $\mathcal{O}$-minimal, $\mathscr{M} \vDash T$ and $p(x)$ a type over $\mathscr{M}$. Then for any $\mathscr{N} \succ \mathscr{M}$, p has at most two coheirs over $\mathscr{N}$.

COROLlaRY 3.10. No strongly O-minimal theory $T$ has the independence property.

Proof of 3.9. Let $p(x)$ be a complete type over $\mathscr{M}$ and let $\mathscr{N} \succ \mathscr{M}$. Let $C(x)$ be the cut over $\mathscr{M}$ determined by $p(x)$, and let $A \subseteq \mathscr{N}$ be the set of elements that satisfy $C(x)$. It is easy to see that if a cut $C^{\prime}(x)$ over $\mathscr{N}$ is to be included in a coheir of $p(x)$, then $C^{\prime}(x)$ must contain either $C^{+}(x)=\{a<x: a \in A\}$ or $C^{-}(x)=$ $\{x<a: a \in A\}$ and, furthermore, that $C^{\prime}(x)$ is completely determined by $C(x) \cup$ $C^{+}(x)$ or $C(x) \cup C^{-}(x)$. Hence, there are at most two cuts that are included in any coheir of $p(x)$ over $\mathscr{N}$. Therefore, by Theorem 3.3, $p(x)$ has at most two coheirs over $\mathscr{N}$. 
The final result of this section completely characterizes those linear orderings in the language $L=\{<\}$ whose theories are strongly 0 -minimal. We first recall some standard notations and a lemma that will be of use. By $\mathscr{M} \equiv_{n} \mathscr{N}$ we shall mean that the structures $\mathscr{M}$ and $\mathscr{N}$ (of the same similarity type) are equivalent for all sentences of quantifier rank less than or equal to $n$. Also, if $\mathscr{M}$ and $\mathscr{N}$ are linear orderings, then $\mathscr{M}+\mathscr{N}$ will denote the ordered sum of $\mathscr{M}$ and $\mathscr{N}$, and, more generally, if $I$ and $\mathscr{M}_{i}, i \in I$, are linear orderings, then $\sum_{i \in I} \mathscr{M}_{i}$ will denote the ordered sum of the set $\left\{\mathscr{M}_{i}: i \in I\right\}$. Lastly, $\omega^{*}$, as usual, represents the reverse ordering on the natural numbers and $\mathbf{Q}$ the ordering of the rational numbers. The following lemma, due to Feferman and Vaught [5], can also be proved using Ehrenfeucht games.

Lemma 3.11. Let $I$ be a linear ordering and $\left\{\mathscr{M}_{i}: i \in I\right\}$ and $\left\{\mathscr{N}_{i}: i \in I\right\}$ sets of linear orderings indexed by $I$. Suppose also that $i_{1}, \ldots, i_{m} \in I$ and, for each $i_{j}$, $j=1, \ldots, m$, that $a_{1}^{i_{j}}, \ldots, a_{p_{j}}^{i_{j}} \in \mathscr{M}_{i}$ and $b_{1}^{i_{j}}, \ldots, b_{p_{j}}^{i_{j}} \in \mathscr{N}_{i_{j}}$. Then, if for each $j=$ $1, \ldots, m,\left(\mathscr{M}_{i_{j}}, a_{1}^{i_{j}}, \ldots, a_{p_{j}}^{i_{j}}\right) \equiv_{n}\left(\mathscr{N}_{i_{j}}, b_{1}^{i_{j}}, \ldots, b_{p_{j}}^{i_{j}}\right)$, it follows that

$$
\left(\sum_{i \in I} \mathscr{M}_{i}, a_{1}^{i_{1}}, \ldots, a_{p_{m}}^{i_{m}}\right) \equiv_{n}\left(\sum_{i \in I} \mathscr{N}_{i}, b_{1}^{i_{1}}, \ldots, b_{p_{m}}^{i_{m}}\right) .
$$

We now can give our characterization of strongly $\mathcal{O}$-minimal linear orderings. Let $\mathscr{F}$ be the set of all finite linear orderings, and $\mathscr{C}=\mathscr{F} \cup\left\{\omega, \omega^{*}, \omega+\omega^{*}, \omega^{*}+\omega, Q\right\}$. Also, let $\mathcal{O}$ be the collection of all ordered sums of the form $C_{1}+\cdots+C_{m}$, where $C_{i}$ is elementarily equivalent to some member of $\mathscr{C}$ for each $i \leqslant m$, and for all $i \leqslant m$, if $C_{i}$ does not have a last element, then $C_{i+1}$ has a first element.

THEOREM 3.12. Any model of a completely strongly O-minimal theory of linear order in the language $\{<\}$ is a member of $\mathcal{O}$, and conversely, the first-order theory of any member of $\mathcal{O}$ is a strongly $\mathcal{O}$-minimal theory of linear order.

The next lemma may be proved by induction on $m$ by using observations such as if $C \equiv \omega+\omega^{*}$ and $D \equiv \omega$, then $C+D \equiv \omega$. Details are left to the reader.

Lemma 3.13. Suppose that $\mathscr{M} \in \mathcal{O}$ and $\mathscr{M}=C_{1}+\cdots+C_{m}$, where for each $i \leqslant m$, $C_{i} \in \mathscr{C}$. If $\mathscr{M}$ has been written using the fewest possible summands from $\mathscr{C}$, then each summand is definable.

Proof of Theorem 3.12. First, suppose that $\mathscr{M} \in \mathcal{O}$. We must show that $\operatorname{Th}(\mathscr{M})$ is strongly $\mathcal{O}$-minimal. Suppose that $\mathscr{M}=C_{1}+\cdots+C_{m}$, where $C_{i}$ is elemientarily equivalent to some member of $\mathscr{C}$ for each $i \leqslant m$ and $m$ is minimal. Lemma 3.13 implies that if $\mathscr{N}=\operatorname{Th}(\mathscr{M}), \mathscr{N}=D_{1}+\cdots+D_{m}$, where $D_{i} \equiv C_{i}$ for each $i \leqslant m$. We must show that any parametrically definable subset of $\mathscr{N}$ is a finite union of intervals in $\mathscr{N}$. By standard arguments (cf. [12], for example) $\mathrm{Th}\left(C_{i}\right)$, for each $i \leqslant m$, admits elimination of quantifiers (in an augmented language, as in [12], for the discrete parts). It then follows-see [12] again for details-that all parametrically definable sets in the linear orderings $D_{i}$, for each $i \leqslant m$, are unions of finitely many intervals. Let $\varphi\left(x, y_{1}^{1}, \ldots, y_{p}^{1}, \ldots, y_{1}^{m}, \ldots, y_{p_{m}}^{m}\right)$ be a formula of quantifier rank $n$ in the language of linear orderings, $a_{1}^{1}, \ldots, a_{p_{1}}^{1} \in D_{1}, \ldots, a_{1}^{m}, \ldots, a_{p_{m}}^{m} \in D_{m}$, and 
$A=\left\{a \in \mathscr{N}: \mathscr{N}=\varphi\left(a, a_{1}^{1}, \ldots, a_{p_{m}}^{m}\right)\right\}$. If $A$ is finite, there is nothing to prove, and so without loss let us suppose that $A$ is infinite. For a contradiction, assume also that $A$ is a discrete set. Without any further loss of generality, $A_{1}=A \cap D_{1}$ may be supposed to be infinite. Since there are only finitely many formulas of quantifier rank less than or equal to $n$ in $p_{1}+1$ free variables, it follows that there is a formula $\psi\left(x, y_{1}, \ldots, y_{p_{1}}\right)$ such that for $b \in D_{1}, D_{1}=\psi\left(b, a_{1}^{1}, \ldots, a_{p_{1}}^{1}\right)$ if and only if there is some $c \in A_{1}$ so that $\left(D_{1}, a_{1}^{1}, \ldots, a_{p_{1}}^{1}, b\right) \equiv_{n}\left(D_{1}, a_{1}^{1}, \ldots, a_{p_{1}}^{1}, c\right)$. Clearly, $B=\left\{b \in D_{1}: D_{1}=\psi\left(b, a_{1}^{1}, \ldots, a_{p_{1}}^{1}\right)\right\}$ contains $A_{1}$, and because $A_{1}$ is discrete and $B$ is a finite union of intervals, it must be the case that $A_{1} \nsubseteq B$. But now, applying Lemma 3.11 , for any $b \in B, \mathscr{N} \vDash \varphi\left(b, a_{1}^{1}, \ldots, a_{p_{m}}^{m}\right)$. The proper containment of $A_{1}$ in $B$ then contradicts the definition of $A_{1}$, and so $A$ could not have been discrete.

For the other direction of the proof, suppose that $\mathscr{M}$ is a model of a strongly $\mathcal{O}$-minimal theory of linear order. We show that $\mathscr{M} \in \mathcal{O}$. Let $P=\{a \in \mathscr{M}: a$ has an immediate predecessor in $\mathscr{M}\}$ and $S=\{a \in \mathscr{M}: a$ has an immediate successor in $\mathscr{M}\}$. It is clear that both $P$ and $S$ are definable subsets of $\mathscr{M}$. Next, we claim that $P \backslash S$ and $S \backslash P$ both are finite. Indeed, were $P \backslash S$ infinite, for example, then strong $\mathcal{O}$-minimality would imply that it contains an infinite interval which obviously is absurd. By strong $\mathcal{O}$-minimality once again, $P \cap S$ consists of finitely many intervals in $\mathscr{M}$, which we may assume are maximal in $P \cap S$. It is not difficult to see then that the elements of $P \cup S \backslash P \cap S$ consist of endpoints of these intervals or the endpoints of discrete intervals consisting of two elements. We thus have that $P \cup S$ consists of finitely many intervals in $\mathscr{M}$, each of which, without difficulty, is seen to be elementarily equivalent to some member of $\mathscr{C} \backslash\{Q\}$. We now examine $\mathscr{M} \backslash P \cup S$, those members of $\mathscr{M}$ that have neither an immediate predecessor nor an immediate successor. Invoking strong $\mathcal{O}$-minimality once more, it follows that $\mathscr{M} \backslash P \cup S$ divides into finitely many intervals, which we suppose are maximal in $\mathscr{M} \backslash P \cup S$. If such an interval is finite, then it readily is seen that it has to be singleton, and if it is infinite, then it must be a dense linear order without endpoints. In either case, the interval is elementarily equivalent to some member of $\mathscr{C}$.

To complete the proof of the theorem, it remains to establish that, when $\mathscr{M}$ is written as the ordered sum of rational intervals as obtained above, (3.12.1) is satisfied. Suppose $\mathscr{M}=C_{1}+\cdots+C_{m}$ when it is so written. It is clear that each $C_{i}$, for $i=1, \ldots, m$, is definable. Condition (3.12.1) then holds by strong $\mathcal{O}$-minimality.

4. Parametrically definable unary functions. The main result in this section is Theorem 4.2, the assertion that every parametrically definable unary function in an $\mathcal{O}$-minimal model is piecewise strictly monotone or constant and continuous. This theorem is the primary tool needed in the proofs of the structure theorem in subsequent sections. As in the illustration of the techniques available under the hypothesis of $\mathcal{O}$-minimality, we first prove the weaker Exchange Principle (cf. the remarks following the statement of Theorem 4.2).

Theorem 4.1 (Exchange Principle for $\mathcal{O}$-minimal models). Let $\mathscr{M}$ be $\mathcal{O}$ minimal. Let $b, c, a_{1}, \ldots, a_{n} \in \mathscr{M}$. If $b$ is algebraic over $c, a_{1}, \ldots, a_{n}$ and $b$ is not algebraic over $a_{1}, \ldots, a_{n}$, then $c$ is algebraic over $b, a_{1}, \ldots, a_{n}$. 
Proof. Since $\operatorname{Th}\left(\mathscr{M}, a_{1}, \ldots, a_{n}\right)$ is strongly $\mathcal{O}$-minimal, we may suppose for ease of notation that $\left\{a_{1}, \ldots, a_{n}\right\}=\varnothing$. Thus suppose that $b$ is algebraic over $c$ and not algebraic over $\varnothing$. Since being algebraic implies being definable, we may assume that there is some parameter-free definable partial function $f$ so that $f(c)=b$.

For a contradiction, suppose that $c$ is not algebraic over $b$. Let $A=\{x \in \mathscr{M}$ : $f(x)=b$ \}. If $c$ were a boundary point of one of the finitely many rational intervals of which $A$ consists, then $c$ would be definable over $b$ and we would be done. Consequently, we may assume that there are $d_{1}, d_{2} \in \mathscr{M} \cup\{ \pm \infty\}$ so that $\left(d_{1}, d_{2}\right)$ $\subseteq A$ and $d_{1}<c<d_{2}$. Moreover, if $\left|\left(d_{1}, d_{2}\right)\right|<\aleph_{0}$, then again $c$ would be definable over $b$. Thus, we also may suppose that $\left|\left(d_{1}, d_{2}\right)\right| \geqslant \boldsymbol{\aleph}_{0}$. Also, if $d_{1}=-\infty$ and $d_{2}=\infty$, then $b$ would be definable over $\varnothing$ (as the unique $y$ in $\mathscr{M}$ such that $\mathscr{M} \vDash \exists x f(x)=y$ ) and hence algebraic over $\varnothing$. So without loss of generality, suppose that $d_{1} \in \mathscr{M}$.

Let $B$ be the definable set given by

$$
\begin{aligned}
& B=\{d \in \mathscr{M}: \mathscr{M} \vDash(“ \exists y \leqslant \infty ") \\
& {[(\exists \geqslant 5 z)(d<z<y)} \\
& \wedge\left(\forall z_{1}\right)\left(\forall z_{2}\right)\left(d<z_{1}<y \wedge d<z_{2}<y \rightarrow f\left(z_{1}\right)=f\left(z_{2}\right)\right) \\
& \wedge \neg(\exists x)(\exists z)((x<d<y \leqslant z \vee x \leqslant d<y<z) \\
& \left.\left.\left.\wedge\left(\forall z_{1}\right)\left(\forall z_{2}\right)\left(x<z_{1}<z \wedge x<z_{2}<z \rightarrow f\left(z_{1}\right)=f\left(z_{2}\right)\right)\right)\right]\right\} .
\end{aligned}
$$

We immediately observe that:

(i) $d_{1} \in B$;

(ii) for every $d \in B$ there is a unique $d^{\prime} \in \mathscr{M} \cup\{\infty\}$ which serves as $y$ in the above formula; and

(iii) if $d, e \in B$ and $d^{\prime}, e^{\prime} \in \mathscr{M} \cup\{\infty\}$ correspond to $d, e$ as in (ii), then $\left(d, d^{\prime}\right) \cap$ $\left(e, e^{\prime}\right)=\varnothing$.

Moreover, we claim that $|B|<\aleph_{0}$. This follows from (iii) and the first conjunct of the formula defining $B$, as otherwise $B$ would be an infinite definable set not containing an infinite interval.

Suppose that $d_{1}$ is the $i$ th member of $B$ in increasing order. But then $b$ is definable without parameters by the formula $\theta(y)$ asserting that " $y$ is the value of $f$ in the interior of the interval determined by (ii) whose left endpoint is the $i$ th member of $B$." With this contradiction to the hypothesis that $b$ is not algebraic, the theorem is proved.

TheOREM 4.2. Let $\mathscr{M}$ be $\mathcal{O}$-minimal and $A \subseteq \mathscr{M}$. Suppose that $f$ is a unary function with domain $(a, b)^{\mathscr{\mu}}$, where possibly $a=-\infty$ and $b=+\infty$, such that $f$ is definable with parameters from $A$, and $a, b$, if different from $\pm \infty$, are elements of $\operatorname{cl}(A)$. Then there are $a_{0}=a, a_{1}, \ldots, a_{n-1}, a_{n}=b \in \mathscr{M} \cup\{ \pm \infty\}$ so that

(a) $a_{0}<a_{1}<\cdots<a_{n}$, and $a_{1}, \ldots, a_{n-1}$ are definable from $A$;

(b) $f$ is monotone or constant on each interval $\left(a_{i-1}, a_{i}\right)^{\mathscr{M}}, i=1, \ldots, n$;

(c) if $f$ is not constant on $\left(a_{i-1}, a_{i}\right)^{\mathscr{\mu}}$, then $f^{\prime \prime}\left(a_{i-1}, a_{i}\right)$ is an interval in $\mathscr{M}$ and $f \uparrow\left(a_{i-1}, a_{i}\right)^{\mathscr{M}}$ is an order preserving or reversing bijection onto $f^{\prime \prime}\left(a_{i-1}, a_{i}\right)^{\mathscr{M}}$.

This theorem may be understood as a strengthening of Theorem 4.1. That is, suppose that $f$ is the parametrically definable partial function such that $f(c)=b$, as 
in the proof of Theorem 4.1. By Theorem 4.2, $f$ is piecewise strictly monotone or constant. The point $c$ either is an endpoint of one of the intervals on which $f$ is monotone or constant, or it is in the interior of one such. In the former case, $c$ is definable over the parameters used to define $f$. Thus suppose that $c$ is in the interior of the $k$ th interval, $I_{k}=\left(a_{k-1}, a_{k}\right)$ on which $f$ is monotone or constant. Clearly, if $f \uparrow I_{k}$ is constant, then $b$ would be definable over $A$, contrary to hypothesis. However, if $f$ is strictly monotone on $I_{k}$, then $f \uparrow I_{k}$ has an inverse, and the following formula, which the reader can verify may be written in first order logic, defines $c$ over $\{b\} \cup A$ :

$$
\text { " } x \in I_{k} \quad \text { and } \quad x=f^{-1}(b) " .
$$

Thus, the Exchange Principle surely follows from Theorem 4.2. The main ingredient in the proof of Theorem 4.2 is the following.

LEMMA 4.3. Let $f$ be a parametrically definable unary function in an O-minimal structure $\mathscr{M}$ with domain $(a, b)^{\mathscr{M}}$, where $a=-\infty, b=+\infty$, or $a$ and $b$ are definable from the parameters used to define $f$. Then, for any parametrically definable infinite interval $I \subseteq(a, b)^{\mu}$, there is an infinite $I^{*} \subseteq I$ on which $f$ is either constant or strictly monotone.

Proof. For ease of notation of language, we shall disregard the parameters used to define $f$ and $I$ and simply speak of each as being definable. The argument will proceed through a case-by-case analysis based on the formulas $\varphi_{0}, \ldots, \varphi_{4}$ below. Let

$$
\begin{gathered}
\varphi_{0}(x) \equiv(\exists z)[(\exists y)(z \leqslant y<x \wedge y \in I \wedge x \in I) \\
\wedge(\forall y)(z \leqslant y<x \rightarrow f(y)=f(x))] \\
\vee(\exists z)[(\exists y)(x<y \leqslant z \wedge y \in I \wedge x \in I) \\
\wedge(\forall y)(x<y \leqslant z \rightarrow f(y)=f(x))] ; \\
\varphi_{1}(x) \equiv(\exists z)(\exists w)[(\exists u)(\exists v)(z \leqslant u<x<v \leqslant w \wedge u \in I \wedge v \in I) \\
\wedge(\forall u)(z \leqslant u<x \rightarrow f(u)<f(x)) \\
\quad \wedge(\forall v)(x<v \leqslant w \rightarrow f(x)<f(v))] ; \\
\varphi_{2}(x) \equiv(\exists z)(\exists w)[(\exists u)(\exists v)(z \leqslant u<x<v \leqslant w \wedge u \in I \wedge v \in I) \\
\wedge(\forall u)(z \leqslant u<x \rightarrow f(u)>f(x)) \\
\quad \wedge(\forall v)(x<v \leqslant w \rightarrow f(x)>f(v))] ; \\
\varphi_{3}(x) \equiv(\exists z)(\exists w)[(\exists u)(\exists v)(z \leqslant u<x<v \leqslant w \wedge u \in I \wedge v \in I) \\
\quad \wedge(\forall u)(z \leqslant u \leqslant w \wedge u \neq x \rightarrow f(u)>f(x))] ;
\end{gathered}
$$

and,

$$
\begin{aligned}
\varphi_{4}(x) \equiv(\exists z)(\exists w)[(\exists u)(\exists v)(z \leqslant u<x<v \leqslant w \wedge u \in I \wedge v \in I) \\
\wedge(\forall u)(z \leqslant u \leqslant w \wedge u \neq x \rightarrow f(u)<f(x))] .
\end{aligned}
$$

Using $O$-minimality, it is evident that $\varphi_{0}, \ldots, \varphi_{4}$ partition $I$ into finitely many rational intervals in the structure $\mathscr{M}$. Thus one of $\varphi_{0}(\mathscr{M})=\left\{a \in \mathscr{M}: \mathscr{M} \vDash \varphi_{0}(a)\right\}$, $\varphi_{1}(\mathscr{M}), \ldots, \varphi_{4}(\mathscr{M})$ must be infinite and hence must contain a definable interval $I_{1} \subseteq I$. 
Case I. $I_{1} \subseteq \varphi_{0}(\mathscr{M})$. Refining $I_{1}$, if necessary, we can assume that for every $x \in I_{1}$, there is $z>x$ so that $(x, z) \neq \varnothing$ and $f \uparrow[x, z)$ is constant. We next assert that

(4.3.1) For some infinite definable $I_{2} \subseteq I_{1}$ and for every $x \in I_{2}$, there are $z<x<w$ so that $[z, x) \neq \varnothing,(x, w] \neq \varnothing$, and $f \uparrow[z, w]$ is constant.

To establish this, it suffices to prove $D=\left\{x \in I_{1}\right.$ : (4.3.1) does not hold for $\left.x\right\}$ is finite, as then $I_{1} \backslash D$ would contain an infinite definable interval $I_{2}$ as required. But $D$ easily is seen not to contain an infinite interval since $D \subseteq I_{1}$ and for any element $x$ of $I_{1}$, there is some $z>x$ so that $(x, z] \neq \varnothing$ and $f \uparrow[x, z]$ is constant. Consequently, by $\mathcal{O}$-minimality, $D$ must be finite. It now follows that $f$ is constant on $I_{2}$. For let $d \in I_{2}$ and $C=\left\{x \in I_{2}: f(d)=f(x)\right\}$. If $C \subsetneq I_{2}$, then, since $C$ is definable using $d$, the $O$-minimality of $\mathscr{M}$ implies that $I_{2}$ contains a boundary point $e$ of $C$. However, $e$ does not satisfy (4.3.1), as is easily seen, and so $f$ must be constant on $I_{2}$.

Case II. $I_{1} \subseteq \varphi_{1}(\mathscr{M})$ or $I_{1} \subseteq \varphi_{2}(\mathscr{M})$. Since the argument is identical for either alternative, we suppose that $I_{1} \subseteq \varphi_{1}(\mathscr{M})$. In this event, we claim that $f$ is strictly monotonically increasing on $I_{1}$. Suppose that $a<b$ and $a, b \in I_{1}$ but $f(a)>f(b)$. Consider the nonempty parametrically definable set $X=\left\{y: y \in I_{1} \wedge y>a \wedge\right.$ $f(a)>f(y)\}$. Since $I_{1} \subseteq \varphi_{1}(\mathscr{M}), X \neq(a, \infty) \cap I_{1}$. So let $c$ be a boundary point in $I_{1}$ of $X \cap I_{1}$. Without loss of generality, suppose that $c$ bounds $X \cap I_{1}$ on the left. But then if $c \in I_{1} \cap X$, then $c$ cannot satisfy $\varphi_{1}(x)$, because for some interval $J$ to the left of $c$, if $x \in J, f(x) \geqslant f(a)>f(c)$. Likewise, if $c \notin I_{1} \cap X$, then since $c$ bounds an interval in $X \cap I_{1}$, for some interval $J$ to the right of $c$, if $x \in J$, then $f(x)<f(c)$. Consequently $c$ could not satisfy $\varphi_{1}(x)$. In any event, $X$ being nonempty leads to an impossibility, and so $f$ must be strictly monotonically increasing, as claimed.

Case III. $I_{1} \subseteq \varphi_{3}(\mathscr{M})$ or $I_{1} \subseteq \varphi_{4}(\mathscr{M})$. Again, the argument for either alternative is the same, so we suppose that $I_{1} \subseteq \varphi_{3}(\mathscr{M})$. Without loss of generality we may assume that $I_{1}$ contains no subinterval-finite or infinite-on which $f$ is constant. That it contains no such infinite subinterval may be assumed since otherwise, the argument would already be done. That it contains no such finite subinterval follows because the $\mathcal{O}$-minimality of $\mathscr{M}$ implies that there can be at most finitely many finite subintervals on which $f$ is constant, and so, if necessary, $I_{1}$ could be cut down. Lastly, we may assume that $I_{1}$ is a dense linear order without endpoints. The density of $I_{1}$ follows because no pair of points $x_{0}$ and $x_{1}$ such that $x_{1}$ is the immediate successor of $x_{0}$ could both satisfy $\varphi_{3}(x)$. That is, it may be supposed that $I_{1}$ does not have endpoints is true, because if necessary $I_{1}$ could be cut down definably so that it does not include endpoints.

We next assert that without loss of generality we may assume that

$$
\left(\forall x \in I_{1}\right)(\exists y)\left(y \in I_{1} \wedge y>x \wedge f(x)>f(y)\right)
$$

and

$$
\left(\forall x \in I_{1}\right)(\exists y)\left(y \in I_{1} \wedge y<x \wedge f(x)>f(y)\right)
$$


To show that we may assume that (4.3.2) holds, consider the definable set

$$
X=\left\{x: x \in I_{1} \wedge(\forall y)\left(y \in I_{1} \wedge y>x \rightarrow f(x) \leqslant f(y)\right)\right\} .
$$

If $X$ is finite, then $I_{1}$ can be refined appropriately. So now, assume that $X$ is infinite and hence contains an infinite definable interval $J$. However, since $I_{1}$ contains no intervals with at least two elements on which $f$ is constant, it then is easy to prove that $f$ is strictly monotonically increasing on $J$, and so we would be done. The argument needed to establish that (4.3.3) holds without loss, is exactly the same.

For any $c \in I_{1},(c, \infty) \cap I_{1}$ can be partitioned into the following three sets:

$$
\begin{aligned}
& X^{+}=\left\{x: x>c \wedge x \in I_{1} \wedge f(x)>f(c)\right\} \\
& X^{=}=\left\{x: x>c \wedge x \in I_{1} \wedge f(x)=f(c)\right\}
\end{aligned}
$$

and

$$
X^{-}=\left\{x: x>c \wedge x \in I_{1} \wedge f(x)<f(c)\right\} .
$$

By $\mathcal{O}$-minimality, this partition consists of finitely many rational intervals in $\mathscr{M}$. We next claim that the rightmost interval, $J$, in this partition is a subset of $X^{-}$. To establish this, we assume that $J \subseteq X^{+}$( $J$ cannot be contained in $X^{=}$since we have assumed that $I_{1}$ contains no interval on which $f$ is constant) and show that this cannot happen by a case-by-case analysis. Let $d \in I_{1}$ be the left-hand boundary point of $J$.

Case A. $d \notin J$, and $d \in X^{-} \cup X^{=}$. In this event, it is evident that $d$ could not satisfy (4.3.2), which is an hypothesis on all of $I_{1}$.

Case B. $d \notin J$ and $d \in X^{+}$. This clearly is impossible as then $d$ would have to be in $J$.

Case C. $d \in J$ and $J \subseteq X^{+}$. To the left of $d$ there must be an interval in $X^{-}$ (again recall $I_{1}$ is dense and contains no interval on which $f$ is constant). In either case, $\varphi_{3}(x)$ again could not hold $d$, contrary to assumption.

Since the cases above exhaust the possibilities, we have shown that $J \subseteq X^{-}$, as asserted. Moreover, since $\mathscr{M} \vDash \varphi_{3}(d)$, it must be true that $d \in J$.

Let

$$
\begin{aligned}
& \varphi_{5}(u) \equiv(\exists z)(\exists w)[(\exists x)(\exists y)(z<x<u<y<w) \\
& \wedge(\exists t)[(\forall s)(s \leqslant u \wedge s<w \rightarrow f(s)<t) \\
&\wedge(\forall s)(s \leqslant u \wedge z<s \rightarrow f(s) \geqslant t)]] .
\end{aligned}
$$

If $c \in I_{1}$ and $d$ is the left-hand boundary point of the rightmost interval in the partition of $(c, \infty) \cap I_{1}$, as above, into $X^{+}, X^{=}$and $X^{-}$, then clearly $\mathscr{M} \vDash \varphi_{5}(d)$. We now claim that $\varphi_{5}(\mathscr{M})$ must be infinite. For, if $\varphi_{5}(\mathscr{M})$ were finite, then we could cut down to an infinite interval $J \subseteq I_{1}$ on which $\neg \varphi_{5}(x)$ holds for each $x \in J$. But then, choosing a point $c \in J$ and its corresponding " $d$ " in $J$, as above, it would follow that $\neg \varphi_{5}(d)$ would have to hold, which is impossible. Consequently let $I_{2} \subseteq I_{1}$ be an infinite definable interval on which $\varphi_{5}(x)$ is satisfied by all $x \in I_{2}$. 
For any $c \in I_{2}$, the same argument as just given shows that there is some $d^{\prime} \in I_{2}$, $d^{\prime}<c$ and some interval $J^{\prime} \subseteq(-\infty, c)$ with $d^{\prime} \in J^{\prime}$ and

$$
\begin{aligned}
& (\forall x)\left(x \in J^{\prime} \wedge x \leqslant d^{\prime} \rightarrow f(x)<f(c)\right) \\
& \left.\wedge(\forall x)\left(x>d^{\prime} \wedge x \in J^{\prime}\right) \rightarrow f(x) \geqslant f(c)\right) .
\end{aligned}
$$

However, it must be true that $\mathscr{M} \vDash \varphi_{5}\left(d^{\prime}\right)$ as well. Let $t$ and $J=(z, w)$ be as guaranteed by $\varphi_{5}\left(d^{\prime}\right)$ holding. We now consider the interval $J^{*}=J \cap J^{\prime}$ about $d^{\prime}$. Without loss, we may assume that $f(x) \neq f(c)$ for all $x \in J^{*}$. Suppose first that $t \geqslant f(c)$. Then $x \in J^{*} \cap\left(-\infty, d^{\prime}\right), f(x) \geqslant t$ since $\varphi_{5}\left(d^{\prime}\right)$ holds. But since (4.3.4) holds, for $x \in J^{*} \cap\left(-\infty, d^{\prime}\right), f(x)<f(c) \leqslant t$, which is impossible. But now, suppose that $t<f(c)$. Then (4.3.4) implies that if $x \in J^{*} \cap\left(d^{\prime}, \infty\right)$, then $f(x) \geqslant$ $f(c)$. However, since $\varphi_{5}\left(d^{\prime}\right)$ holds, $f(x)<t<f(c)$ for any $x \in J^{*} \cap\left(d^{\prime}, \infty\right)$. This again is impossible, and so we have reached a contradiction under the assumption that $\varphi_{3}(\mathscr{M})$ is infinite. This completes the proof of Lemma 4.3.

Now we can finish the proof of Theorem 4.2.

Proof of TheOrem 4.2. Let $\theta(x)$ be the formula asserting that:

"On an interval of which $x$ is the left endpoint, $f$ is strictly monotone or constant, and there is no interval extending this interval on the left on which $f$ is strictly monotone or constant."

It is an elementary exercise to write out $\theta(x)$ in first order logic. We now claim that $\theta(\mathscr{M})$ must be finite. Indeed, were it infinite, it would contain an infinite interval $I$. But by Lemma 4.3, there is an infinite interval $I^{*} \subseteq I$ on which $f$ is strictly monotone or constant. However, any interior point of $I^{*}$ could not satisfy $\theta(x)$, whence we see that $\theta(\mathscr{M})$ must be finite.

Thus, let $\theta(\mathscr{M})=\left\{b_{1}, \ldots, b_{k-1}\right\}$ be enumerated in increasing order, and let $b_{0}=-\infty$ and $b_{k}=\infty$. We claim that $f$ is strictly monotone or constant on each $\left(b_{j-1}, b_{j}\right)$ for $j=1, \ldots, k$. Now $b_{j-1}$, by virtue of satisfying $\theta(x)$, is the left endpoint of an interval on which $f$ is strictly monotone or constant. Let $I \subseteq\left(b_{j-1}, b_{j}\right)$ be the largest such interval. If $\left(b_{j-1}, b_{j}\right) \backslash I \neq \varnothing$ but is finite, then clearly some member of $\left(b_{j-1}, b_{j}\right) \backslash I$ would satisfy $\theta(x)$, which is impossible. But if $\left(b_{j-1}, b_{j}\right) \backslash I$ were infinite, then Lemma 4.3 would imply the existence of an infinite interval $J \subseteq$ $\left(b_{j-1}, b_{j}\right) \backslash I$ on which $f$ is strictly monotone or constant. Again, however, this would imply that $\theta(\mathscr{M}) \cap\left(b_{j-1}, b_{j}\right) \neq \varnothing$, which cannot be true. Therefore $I=$ $\left(b_{j-1}, b_{j}\right)$, as claimed.

Suppose now that $f \uparrow\left(b_{j-1}, b_{j}\right)$ is strictly monotone. The $\mathcal{O}$-minimality of $\mathscr{M}$ implies that $f^{\prime \prime}\left(b_{j-1}, b_{j}\right)$ is the union of finitely many intervals in $\mathscr{M}$. Using the strict montonicity of $f$ on $\left(b_{j-1}, b_{j}\right)$, we can subdivide $\left(b_{j-1}, b_{j}\right)$ into finitely many intervals with appropriately chosen endpoints $b_{j-1}=b_{0}^{j}<b_{1}^{j}<\cdots<b_{k_{j}}^{j}=b_{j}$ such that $f^{\prime \prime}\left(b_{r-1}^{j}, b_{r}^{j}\right)$ is an interval and $f \uparrow\left(b_{r-1}^{j}, b_{r}^{j}\right)$ is an order preserving or reversing bijection onto $f^{\prime \prime}\left(b_{r-1}^{j}, b_{r}^{j}\right)$ for $r=1, \ldots, k_{j}$. 
Finally, if $\left\{a_{0}, \ldots, a_{n}\right\}$ enumerates in increasing order all endpoints as obtained as above, i.e.,

$$
\left\{a_{0}, \ldots, a_{n}\right\}=\left\{b_{0}, \ldots, b_{k}\right\} \cup \bigcup_{j}\left\{b_{0}^{j}, \ldots, b_{k_{j}}^{j}\right\}, \quad f \uparrow\left(b_{j-1}, b_{j}\right) \text { monotone, }
$$

we see that (a)-(c) in the conclusion of Theorem 4.2 are satisfied.

5. Existence and uniqueness of prime models. Throughout this section, we shall adopt the convention that in any context in which a theory $T$ has been fixed, all models will be elementary submodels of some sufficiently saturated model of $T$. Recall that if $A \subseteq \mathscr{M} \vDash T$, then a model $\mathscr{M} \vDash T$ such that $A \subseteq \mathscr{M}$ is said to be prime over $A$ if for any $\mathscr{M}^{\prime} \vDash T$ with $A \subseteq \mathscr{M}^{\prime}$, there is an elementary mapping $f$ : $\mathscr{M} \rightarrow \mathscr{M}^{\prime}$ that is the identity on $A$. With $T$ the theory of real closed fields, for example, the real closure $R$ of an ordered field $F$ is prime over $F$. Moreover, in this case, $R$ also is unique up to isomorphism over $F$. We generalize the existence and uniqueness of the real closure of an ordered field with the following theorem, whose proof is the concern of this section.

TheOREM 5.1. Let $A \subseteq \mathscr{M} \vDash T$, where $T$ is a strongly O-minimal theory. Then there is an $\mathscr{M} \vDash T, A \subseteq \mathscr{M}$, that is prime over $A$, and is unique up to isomorphism over $A$.

The proof of Theorem 5.1 crucially depends on Lemma 5.2. We must make a definition before stating the lemma. Let $A, B \subseteq \mathscr{M}$, where $\mathscr{M}$ is $O$-minimal. The Exchange Principle (Theorem 4.1) permits the definition of the dimension of $B$ over $A$, which we write as $\operatorname{dim}_{A}(B)$, as the cardinality of any set of elements of $B$ that is maximal with respect to being algebraically independent over $A$.

LemMA 5.2. Let $A \subseteq \mathscr{N} \vDash T$, where $T$ is strongly O-minimal, and $a, b \in \operatorname{cl}(A)$ such that the formula $a<x<b$ isolates a complete type over $A$. Then there exists $\mathscr{M} \vDash T$ such that $A \subseteq \mathscr{M}$ and $\operatorname{dim}_{A}(\{c \in \mathscr{M}: a<c<b\}) \leqslant \aleph_{0}$.

The proof of Theorem 5.1 from Lemma 5.2 will be given now, and the proof of Lemma 5.2 will be postponed until later in this section. We first will have to avail ourselves of some preliminary definitions and lemmas.

Let $A \subseteq B \subseteq \mathscr{M}$. Then $B$ is said to be atomic over $A$ provided that for every $n<\omega$, every $n$-tuple of elements from $B$ realizes a principal type over $A$ (in $\mathscr{M}$ ). Moreover, if $B \backslash A$ can be enumerated as a sequence $\left\langle b_{\beta}: \beta<\alpha\right\rangle$, for some ordinal $\alpha$, so that each element $b_{\beta}$ realizes (in $\mathscr{M}$ ) a principal type over $A \cup\left\{b_{\gamma}: \gamma<\beta\right\}$, then $B$ is said to be constructible over $A$. Observe that if $B$ is constructible over $A$, then $B$ is atomic over $A$. The next lemma is well known.

LEMMA 5.3. Let a complete theory $T$ be given.

(a) Let $A \subseteq \mathscr{M} \vDash T$. Any model $\mathscr{M}$ that is constructible over $A$ also is prime over $A$.

(b) Suppose that for any subset $A$ of a model $\mathscr{M}$ of $T$ and any formula $\varphi$ having parameters from $A$, there is a complete formula with parameters from $A$ which, relative to $\operatorname{Th}(\mathscr{M}, a)_{a \in A}$, implies $\varphi$. Then, for any $A \subseteq \mathscr{M}$, there is a model $\mathscr{M}$ of $T$ that is constructible over $A$. 
(c) (Ressayre, unpublished; see IX.4.12 in [0] for a proof.) Let $A \subseteq \mathscr{M} \vDash T$. Then any two models that are constructible over $A$ are isomorphic over $A$.

By Lemma 3.1, it follows from Lemma 5.3(b) that there exists a constructible model over any subset $A$ of a model of strongly $O$-minimal theory $T$. By 5.3(a), any such constructible model will be prime over $A$. Hence, by 5.3(c), we will have established Theorem 5.1 once we prove the next lemma.

Lemma 5.4. Let $\mathscr{M}$ be a model of a strongly O-minimal theory $T$ that is prime over a subset $A$ of a model of $T$. Then $\mathscr{M}$ is constructible over $A$.

We shall require two more lemmas for the proof of Lemma 5.4.

Lemma 5.5. Let $T$ be strongly O-minimal, $A \subseteq \mathscr{M} \vDash T$ and $a, b \in \operatorname{cl}(A)$.

(a) Suppose that the formula " $a<x<b$ " isolates a complete nonalgebraic type over A. Then $I=\{c \in \mathscr{M}: a<c<b\}$ either is a discrete linear order without endpoints, or a dense linear order without endpoints.

(b) Suppose that $I$ is as above, and $I^{\prime}$ is any other open interval in $\mathscr{M}$ all of whose elements satisfy the same nonalgebraic principal type over $A$. If for some $b \in I$, $\mathrm{cl}_{A}(\{b\}) \cap I^{\prime} \neq \varnothing$, then there exists a monotone bijection $g: I \rightarrow I^{\prime}$ that is definable with parameters from $A$. Moreover, any $f: I \rightarrow I$ that is definable over $A$ must be $a$ monotone increasing bijection.

(c) Suppose that $I$ and $f: I \rightarrow I^{\prime}$ are as in (b), and $X \subseteq I$ is atomic and algebraically independent over $A$. Then $f^{\prime \prime}(X)=\{f(x): x \in X\}$ is an atomic and algebraically independent subset of $I^{\prime}$. Furthermore, if $A$ is maximal, then so is $f^{\prime \prime}(X)$.

(d) Let $I$ and $I^{\prime}$ be as above, and $\left\{b_{1}, \ldots, b_{n+1}\right\}$ is an atomic and algebraically independent subset of $I$. Suppose also that $\mathrm{cl}_{A}\left(\left\{b_{1}, \ldots, b_{n}\right\}\right) \cap I^{\prime}=\varnothing$, but $\operatorname{cl}_{A}\left(\left\{b_{1}, \ldots, b_{n+1}\right\}\right) \cap I^{\prime} \neq \varnothing$. Then there is a $J \subseteq I$ such that $b_{n+1} \in J$ and every element of $J$ satisfies the same principal type of $A \cup\left\{b_{1}, \ldots, b_{n}\right\}$, and a monotone bijection $g: J \rightarrow I^{\prime}$ that is definable from parameters in $A \cup\left\{b_{1}, \ldots, b_{n}\right\}$.

Proof. Notice first that since $a, b \in \operatorname{cl}(A)$, and so are definable over $A$, that the formula " $a<x<b$ " can be taken as a formula over $A$. The verification of (a), then, is quite simple. Suppose that $I$ is not discrete, and so some $a \in I$ has, without loss of generality, no immediate successor in $I$. Then $a$ cannot have an immediate predecessor, since any predecessor $b$ of $a$ must satisfy the same type over $A$ as $a$, and thus could not have an immediate successor. Since every element of $I$ satisfies the same type over $A$ as $a$, no element of $I$ can have an immediate successor or predessor and, consequently, $I$ must be a dense linear order without endpoints.

Now we prove (b). Suppose then that $\varphi(x, b)$ is an algebraic formula with (suppressed) parameters from $A$ such that for some $c \in I^{\prime}, \mathscr{M} \vDash \varphi(c, b)$. Since $\mathrm{cl}_{A}(\{b\})=\operatorname{dcl}_{A}(\{b\})$, we also may assume that

$$
\mathscr{M} \vDash(\exists ! x) \varphi(x, b) \wedge \text { “ } x \in I^{\prime} . "
$$

Since the formula $(\exists ! x) \varphi(x, y) \wedge$ " $x \in I^{\prime \prime}$ " has parameters from $A$, it follows that

$$
\mathscr{M} \vDash(\exists ! x) \varphi\left(x, b^{\prime}\right) \wedge \text { “ } x \in I^{\prime} "
$$


for any $b^{\prime} \in I$. Let $f: I \rightarrow I^{\prime}$ be the function defined with parameters from $A$ by $f(b)=c$ if and only if $\mathscr{M} \vDash \varphi(c, b)$. We show that $f$ must be a monotone bijection. By Theorem 4.2, $f$ must be piecewise monotone or constant. However, $f$ cannot change its behavior, because a boundary point of such a change would be an interior point of $I$ that is definable over $A$. Thus, $f$ has to be monotone or constant. If $f$ were constant, though, the range of $f$ would then be a point in $I^{\prime}$ definable over $A$, whence $f$ has to be monotone. Lastly, since the range of $f$ is definable over $A$, it is apparent that the range of $f$ must be all of $I^{\prime}$. We therefore have shown that $f$ is a monotone bijection, as desired. Lastly, it remains to show that $g: I \rightarrow I$ must be a monotonically increasing bijection. This follows simply because were $f$ decreasing, then both $D^{+}=\{b \in I: f(b) \geqslant b\}$ and $D^{-}=\{b \in I: f(b)<b\}$ would be nonempty subsets of $I$ that are definable over $A$ and so would yield a boundary point in $I$ that also would be definable over $A$.

Using (b), the proof of (c) becomes routine and will be left to the reader. So it remains to establish (d). Since $\operatorname{cl}_{A}\left(\left\{b_{1}, \ldots, b_{n}\right\}\right) \cap I^{\prime}=\varnothing$, we conclude that every element of $I^{\prime}$ satisfies the same nonalgebraic principal type over $A \cup\left\{b_{1}, \ldots, b_{n}\right\}$. Moreover, since $\left\{b_{1}, \ldots, b_{n+1}\right\}$ is atomic and independent over $A$, it follows that there is some open $J \subseteq I$ so that $b_{n+1} \in J$ and every element of $J$ satisfies the same nonalgebraic principal type over $A \cup\left\{b_{1}, \ldots, b_{n}\right\}$. Applying (b) to $J$ and $I^{\prime}$ then yields the desired conclusion, completing the proof of 5.5.

A proof of the following lemma may be found in [17].

Lemma 5.6 (HARrington). Let $T$ be a complete theory. For any subset $A$ of a model of $T$, suppose also that every formula with parameters from $A$ is implied by a complete formula with parameters from $A$. Then, if $B \subseteq C \subseteq D$ all are submodels of a model of $T, D$ is atomic over $B$, and for any complete type over $B$, either all or none of its realizations in $D$ are in $C$, it follows that $D$ is atomic over $C$.

Now we can proceed with the proof of Lemma 5.4. Here, we shall avail ourselves of Lemma 5.2.

Proof of Lemma 5.4. Let $\mathscr{M} \vDash T$ be prime over $A$. Let $\left\langle I_{\alpha}: \alpha<\lambda\right\rangle$ enumerate all open intervals in $\mathscr{M}$ having the property that all elements in the interval satisfy the same nonalgebraic principal type over $A$. Observe that $\mathscr{M}=\operatorname{cl}(A) \cup \cup_{\alpha<\lambda} I_{\alpha}$. Now, any such $I_{\alpha}$ consists of the set of elements in $\mathscr{M}$ satisfying the formula " $a_{\alpha}<x<b_{\alpha}$ ", for some $a_{\alpha}, b_{\alpha} \in \operatorname{cl}(A)$. By Lemma 5.2, there exists a model $N_{\alpha}$ containing $A$ in which $\operatorname{dim}_{A}\left(\left\{c \in N_{\alpha}: N_{\alpha} \vDash a_{\alpha}<c<b_{\alpha}\right\}\right)$ is countable. Since $\mathscr{M}$ is prime over $A$, we can elementarily embed $\mathscr{M}$ into $N_{\alpha}$ over $A$, from which it follows that $\operatorname{dim}_{A}\left(I_{\alpha}\right) \leqslant \boldsymbol{\aleph}_{0}$. For each $\alpha<\lambda$, we now fix an enumeration $C_{\alpha}=\left\langle c_{n}^{\alpha}: n<\omega\right\rangle$ of a maximal algebraically independent and (since $\mathscr{M}$ is prime and hence atomic) atomic subset of $I_{\alpha}$. We now show that $\mathscr{M}$ is constructible.

We shall enumerate $\mathscr{M} \backslash A$ as the concatenation of a recursively defined sequence of sequences of elements of $\mathscr{M},\left\langle D_{\alpha}: \alpha<\lambda\right\rangle$, which will be seen to be a construction. Moreover, we will show, for each $\alpha<\lambda$, that

(5.4.1) $I_{\alpha} \subseteq \cup_{\beta \leqslant \alpha} D_{\beta}$.

(5.4.2) $\cup_{\beta \leqslant \alpha} D_{\beta} \cup A$ is algebraically, and, hence, definably closed, and

(5.4.3) for each $\gamma<\lambda$, if $I_{\gamma} \nsubseteq \cup_{\beta \leqslant \alpha} D_{\beta}$, then $I_{\gamma} \cap \cup_{\beta \leqslant \alpha} D_{\beta}=\varnothing$. 
We now build $\left\langle D_{\alpha}: \alpha<\lambda\right\rangle$.

First, we enumerate $D_{0}$. Let $\operatorname{cl}(A) \backslash A=\operatorname{dcl}(A) \backslash A$ be enumerated as $F_{0}=\left\langle f_{\gamma}: \gamma\right.$ $\left\langle\beta_{0}\right\rangle$. It is obvious that $F_{0}$ constitutes a construction over $A$. Notice that $F_{0} \cap I_{\alpha}$ $=\varnothing$ for all $\alpha<\lambda$ and thus that each element of any given $I_{\alpha}$ satisfies the same principal type over $A \cup F_{0}$. Since $\mathscr{M}$ is atomic over $A$, clearly $\mathscr{M}$ is atomic over $A \cup F_{0}$. Therefore, since the order type of $C_{0}=\left\langle c_{n}^{0}: n\langle\omega\rangle\right.$ is $\omega$, it is a routine matter to verify that $C_{0}$ is a construction over $A \cup F_{0}$. Next, let $E_{0}=\left\langle e_{\nu}^{0}: \nu\left\langle\delta_{0}\right\rangle\right.$ enumerate $\mathrm{cl}_{A}\left(F_{0} \cup C_{0}\right) \backslash A \cup F_{0} \cup C_{0}$. Again, obviously $E_{0}$ is a construction over $A \cup F_{0} \cup C_{0}$. Finally, let $D_{0}=F_{0} \frown C_{0} \frown E_{0}$. Observe, first, by the maximality of $C_{0}$, that $I_{0} \subseteq D_{0}$. Furthermore, $D_{0} \cup A$ is algebraically closed. Lastly, by Lemma $5.5(\mathrm{c})$, (d), for any $\gamma<\lambda$, if $I_{\gamma} \nsubseteq D_{0}$, then $I_{\gamma} \cap D_{0}=\varnothing$. Hence (5.4.1)-(5.4.3) hold for $\alpha=0$.

Next, suppose that (5.4.1)-(5.4.3) hold for all $\beta<\alpha$. We build $D_{\alpha}$ so that they continue to be satisfied. Observe that $\cup_{\beta<\alpha} D_{\beta} \cup A$ is algebraically closed and also, for each $\gamma<\lambda$, if $I_{\gamma} \nsubseteq \cup_{\beta<\alpha} D_{\beta}$, then $I_{\gamma} \cap \bigcup_{\beta<\alpha} D_{\beta}=\varnothing$. For any $\gamma<\lambda$, it then follows that either $I_{\gamma} \subseteq \bigcup_{\beta<\alpha} D_{\beta}$ or $I_{\gamma} \cap \bigcup_{\beta<\alpha} D_{\beta}=\varnothing$, and in the latter event that each member of $I_{\gamma}$ satisfies the same nonalgebraic principal type over $A \cup \cup_{\beta<\alpha} D_{\beta}$ (recall that the endpoints of $I_{\gamma}$ are in $\operatorname{cl}(A)$ ). This fact, together with Lemma 3.1, suffices to show that $A \subseteq A \cup \cup_{\beta<\alpha} D_{\beta} \subseteq \mathscr{M}$ satisfy the hypothesis of Lemma 5.6. Hence $\mathscr{M}$ is atomic over $A \cup \cup_{\beta<\alpha} D_{\beta}$. If $I_{\alpha} \subseteq \cup_{\beta<\alpha} D_{\beta}$, then let $D_{\alpha}=\langle\rangle$, in which case (5.4.1)-(5.4.3) trivally continue to hold. Thus assume that $I_{\alpha} \cap \bigcup_{\beta<\alpha} D_{\beta}$ $=\varnothing$. Since $C_{\alpha}=\left\langle c_{n}^{\alpha}: n\langle\omega\rangle\right.$ has order type $\omega$, it of course is a construction over $A \cup \cup_{\beta<\alpha} D_{\beta}$. Next, let $E_{\alpha}=\left\langle e_{\nu}^{\alpha}: \nu<\delta_{\alpha}\right\rangle$ enumerate $\mathrm{cl}_{A}\left(\cup_{\beta<\alpha} D_{\beta} \cup C_{\alpha}\right)$. Again, $E_{\alpha}$ constitutes a construction over $A \cup \cup_{\beta<\alpha} D_{\beta}$. Finally, let $\left.D_{\alpha}=C_{\alpha}\right\urcorner E_{\alpha}$. Since $C_{\alpha}$ is a maximal independent subset of $I_{\alpha}$ over $A$, we have that $I_{\alpha} \subseteq \cup_{\beta<\alpha} D_{\beta}$, so that (5.4.1) is satisfied. Nothing needs to be said about the continued satisfaction of (5.4.2). Lastly, (5.4.3) continues to hold by virtue of Lemma 5.5(c), (d). This completes the definition of $\left\langle D_{\alpha}: \alpha<\lambda\right\rangle$, which obviously serves as a construction of $\mathscr{M}$ over $A$, and so finishes the proof of Lemma 5.4.

The proof of Theorem 5.1 therefore will be complete once Lemma 5.2 has been proved. We shall need a lemma.

Lemma 5.7. Suppose that $A \subseteq \mathscr{N}=T$, where $T$ is strongly O-minimal, $a, b \in \operatorname{cl}(A)$ and that the formula " $a<x<b$ " isolates a nonalgebraic complete type over A. Also assume that $X \subseteq I=(a, b)^{\mathcal{N}}$ and that $\mathrm{cl}_{A}(X) \cap I$ is dense if $I$ is dense, or discrete if $I$ is discrete (cf. Lemma 5.5(a)). Then, if $\mathscr{M}$ is atomic over $\mathrm{cl}_{A}(X)$, it follows that $(a, b)^{\mathscr{M}} \subseteq \mathrm{cl}_{A}(X)$.

Proof. Let $c \in(a, b)^{\mathscr{\mu}}$. Then $c$ satisfies an atomic type over $\mathrm{cl}_{A}(X)$. If this type is algebraic, then clearly $c \in \mathrm{cl}_{A}(X)$. On the other hand the assumption that the type that $c$ satisfies over $\operatorname{cl}_{A}(X)$ is nonalgebraic and thus of the form " $e_{1}<x<e_{2}$ " for some $e_{1}, e_{2} \in \mathrm{cl}_{A}(X)$ leads to a contradiction. Indeed, our hypothesis, in the case that $\left(e_{1}, e_{2}\right)^{\mathscr{N}}$ is either dense or discrete (and infinite), implies that $\left(e_{1}, e_{2}\right)^{\mathscr{N}} \cap$ $\mathrm{cl}_{A}(X) \neq \varnothing$. But then it cannot be the case that " $e_{1}<x<e_{2}$ " isolates a complete 
type over $\mathrm{cl}_{A}(X)$, contrary to what we supposed. The proof of the lemma now is complete.

The conclusion of the proof of the following lemma is due to David Marker. It greatly simplifies our original argument and is very much in the spirit of the kind of argument that we had sought but were not successful in finding.

Lemma 5.2. Let $A \subseteq \mathscr{N} \vDash T$, where $T$ is strongly O-minimal, and $a, b \in \operatorname{cl}(A)$ such that the formula $a<x<b$ isolates a complete type over $A$. Then there exists $\mathscr{M} \vDash T$ such that $A \subseteq \mathscr{M}$ and $\operatorname{dim}_{A}(\{c \in \mathscr{M}: a<c<b\}) \leqslant \boldsymbol{\aleph}_{0}$.

Proof. By Lemmas 3.1 and 5.3(b), there exists a constructible model over any subset of a model of $T$. Since any model that is constructible over $A$ also is atomic over $A$, by applying Lemma 5.7 to a model $\mathscr{M}$ that is constructed over $\operatorname{cl}(A)$, we shall be done once we have found some $X \subseteq(a, b)^{\mathcal{N}}$ so that $\operatorname{cl}_{A}(X)$ is dense or discrete if $(a, b)^{\mathcal{N}}$ is and $\operatorname{dim}_{A}(X) \leqslant \boldsymbol{\aleph}_{0}$.

If $(a, b)^{\mathscr{N}}$ is discrete, then simply let $X$ be any singleton. We thus may assume that $(a, b)^{\mathscr{N}}$ is a dense linear order without endpoints. We construct $X$ as the union of an increasing chain of finite sets $X_{n}, n<\omega$. For notational convenience let $X_{-1}=\varnothing$.

At stage $n=0$, choose some $x_{0} \in(a, b)^{\mathcal{N}}$ and let $X_{0}=\left\{x_{0}\right\}$. Also, let $\mathscr{J}_{0}$ enumerate in some fixed order all nonalgebraic principal types over $\mathrm{cl}_{A}\left(X_{0}\right)$ with an endpoint in $X_{0}$ (e.g., formulas of the form " $c<x<x_{0}$ " or " $x_{0}<x<c$," where $\left.c \in \mathrm{cl}_{A}\left(X_{0}\right)\right)$.

Now suppose that we have the sets $X_{i}, i<n$, and $\mathscr{J}_{i}, i<n$. The construction of $X_{n}$ now breaks up into cases:

Case 1. There is no member of $\bigcup_{i<n} \mathscr{J}_{i}$ that is still isolated over $\mathrm{cl}_{A}\left(\cup_{i<n} X_{i}\right)$. In this case, let $X_{n}=X_{n-1}$ and $\mathscr{J}_{n}=\langle\rangle$.

Case 2. There is some member of $\bigcup_{i<n} \mathscr{J}_{i}$ that is still isolated over $\mathrm{cl}_{A}\left(\mathrm{U}_{i<n} X_{i}\right)$. Let $J$ be the least such member of the enumeration $\mathscr{J}_{0} \wedge \mathscr{J}_{1}^{\wedge} \ldots \wedge \mathscr{J}_{n-1}$, and choose $x_{n} \in J$. Then let $X_{n}=X_{n-1} \cup\left\{x_{n}\right\}$, and let $\mathscr{J}_{n}$ be an enumeration of all nonalgebraic principal types over $\operatorname{cl}_{A}\left(X_{n}\right)$ that have an endpoint in $X_{n}$.

Setting $X=\cup_{n<\omega} X_{n}$, we now assert that $\operatorname{cl}_{A}(X) \cup(a, b)^{\mathscr{N}}$ is dense. Indeed, suppose that $c, d \in \mathrm{cl}_{A}(X) \cap(a, b)^{\mathcal{N}}, c<d$, and let $n$ be least such that $c, d \in$ $\operatorname{cl}_{A}\left(X_{n}\right)$. Without loss of generality, we may assume that $c \in \operatorname{cl}_{A}\left(X_{n}\right) \backslash \operatorname{cl}_{A}\left(X_{n-1}\right)$, and so $X_{n}=X_{n-1} \cup\left\{x_{n}\right\}$. Let the formula " $e_{1}<x<e_{2}$ " isolate the complete type over $A \cup X_{n-1}$ that $x_{n}$ realizes. As usual, there exists a function $f$ with domain $\left(e_{1}, e_{2}\right)^{\mathcal{N}}$ that is definable from the parameters $A \cup X_{n-1}$ such that $f\left(x_{n}\right)=c$.

Since " $e_{1}<x<e_{2}$ " isolates a complete type over $A \cup X_{n-1}, f$ must be monotone or constant on $\left(e_{1}, e_{2}\right)^{\mathcal{N}}$. But since $c \in \mathrm{cl}_{A}\left(X_{n}\right) \backslash \mathrm{cl}_{A}\left(X_{n-1}\right), f$ must be monotone on $\left(e_{1}, e_{2}\right)^{\mathcal{N}}$. Also, we may infer that $R=f^{\prime \prime}\left(\left(e_{1}, e_{2}\right)^{\mathcal{N}}\right)$ must be an interval in $\mathscr{N}$, again because every element of $\left(e_{1}, e_{2}\right)^{\mathcal{N}}$ satisfies the same type over $A \cup X_{n-1}$. Thus $f$ is an order preserving or reversing bijection from $\left(e_{1}, e_{2}\right)^{\mathscr{N}}$ onto $R=\left(e_{1}^{\prime}, e_{2}^{\prime}\right)^{\mathcal{N}}$, and $c$ is an interior point of $\left(e_{1}^{\prime}, e_{2}^{\prime}\right)^{\mathcal{N}}$. Without loss of generality, we suppose that $f$ is increasing. 
If $e_{2}^{\prime}<d$, then since we would have $c<e_{2}^{\prime}<d$ and $e_{2}^{\prime}$ already is definable over $A \cup X_{n-1}$, we would be done. Hence we further suppose that $d \leqslant e_{2}^{\prime}$. Therefore,

$$
\begin{aligned}
\operatorname{cl}_{A}(X) \cap(c, d)^{\mathscr{N}} \neq \varnothing \\
\quad \operatorname{iff} \begin{cases}\operatorname{cl}_{A}(X) \cap\left(x_{n}, f^{-1}(d)\right)^{\mathcal{N}} \neq \varnothing & \text { if } d<e_{2}^{\prime}, \\
\operatorname{cl}_{A}(X) \cap\left(x_{n}, e_{2}\right)^{\mathcal{N}} \neq \varnothing & \text { if } d=e_{2}^{\prime}\end{cases}
\end{aligned}
$$

If there is no nonalgebraic principal type over $\mathrm{cl}_{A}\left(X_{n}\right)$ with left endpoint $x_{n}$, then obviously $\mathrm{cl}_{A}\left(X_{n}\right) \cap(c, d)^{\mathscr{N}} \neq \varnothing$. On the other hand, suppose there exists such a type given by the interval $I$. It follows that

$$
I \subseteq \begin{cases}\left(x_{n}, f^{-1}(d)\right)^{\mathcal{N}} & \text { if } d<e_{2}^{\prime} \\ \left(x_{n}, e_{2}\right)^{\mathcal{N}} & \text { if } d=e_{2}^{\prime}\end{cases}
$$

and by some stage in the construction either $I$ no longer is isolated, or a point chosen from $I$ is put into $X$. In either event, $\operatorname{cl}_{A}(X) \cap I \neq \varnothing$, whence the proof is complete.

6. $\aleph_{0}$-categorical strongly 0 -minimal theories. Here, we prove the following theorem, which completely characterizes those strongly 0 -minimal theories that are $\aleph_{0}$-categorical.

THEOREM 6.1. Let $T$ be an $\boldsymbol{\aleph}_{0}$-categorical strongly O-minimal theory, and let $\mathscr{M}$ be its model (up to isomorphism) of power $\aleph_{0}$. Then there exist

(i) a finite $C=\left\{c_{0}, \ldots, c_{n}\right\} \subseteq \mathscr{M}(\mathscr{M} \cup\{ \pm \infty\}$, if $\mathscr{M}$ does not have a first or last element), consisting of all of the definable elements in $\mathscr{M}$ (with the possible exceptions of $\pm \infty)$, such that $\mathscr{M} \vDash c_{i}<c_{j}$ for all $i<j \leqslant n$ and for each $j=1, \ldots, n$, either $\mathscr{M} \vDash \neg(\exists x) c_{j-1}<x<c_{j}$ or $I_{j}=\left\{x \in \mathscr{M}: \mathscr{M} \vDash c_{j-1}<x<c_{j}\right\}$ is a dense linear order without endpoints and

(ii) an equivalence relation $E \subseteq\left(\left\{j: I_{j} \neq \varnothing\right\}\right)^{2}$ such that for each $(i, j) \in E$ there is a unique definable monotone bijection $f_{i, j}: I_{i} \rightarrow I_{j}$ so that $f_{i, i}=$ identity and $f_{j, k} \circ f_{i, j}$ $=f_{i, k}$ for all $(i, j),(j, k) \in E$,

so that $T$ admits elimination of quantifiers down to the language $\{=,<\} \cup\left\{\underline{c}_{i}\right.$ : $i \leqslant n\} \cup\left\{\underline{f}_{i, j}:(i, j) \in E\right\}$, where the $\underline{c}_{i}, i=0, \ldots, n$, are interpreted in $\mathscr{M}$ by $c_{i},{ }^{4}$ and $\underline{f}_{i, j}$ by $f_{i, j}$ for $(i, j) \in E$. Moreover to any ordering with distinguished elernents as in (i) and any suitable equivalence relation $E$ as in (ii), there corresponds an $\boldsymbol{\aleph}_{0}$-categorical strongly O-minimal theory as above.

As an immediate corollary, we have

COROLlary 6.2. Any $\boldsymbol{\aleph}_{0}$-categorical strongly 0 -minimal theory in a finite language is finitely axiomatizable.

\footnotetext{
${ }^{4}$ Adjustments must be made here if $\mathscr{M}$ does not have a first or last element.
} 
The proof of the "moreover" clause in Theorem 6.1 is not difficult and is left to the reader. The proof of the first part of Theorem 6.1 occupies the rest of this section. The next lemma is standard.

LEMMA 6.3. Any complete expansion by definitions or finitely many constants of an $\aleph_{0}$-categorical theory is again $\boldsymbol{\aleph}_{0}$-categorical.

For the remainder of this section, we fix an $\boldsymbol{\aleph}_{0}$-categorical strongly $\boldsymbol{O}$-minimal theory $T$ and its unique, up to isomorphism, countable model $\mathscr{M}$. We prove a sequence of lemmas that will culminate in the proof of Theorem 6.1.

LEMMA 6.4. The finite set $C$ asserted to exist in (i) of 6.1 does exist.

Proof. Let $C=\{c \in \mathscr{M}: c$ is definable in $\mathscr{M}\}$. By Ehrenfeucht's characterization of $\boldsymbol{\aleph}_{0}$-categoricity, $C$ must be finite. Let $C(\cup\{ \pm \infty\}$ if $\mathscr{M}$ does not have a first or last element) be enumerated as $\left\{c_{0}, \ldots, c_{n}\right\}$. Next, suppose that $I_{j}=\{x \in \mathscr{M}$ : $\left.\mathscr{M} \vDash c_{j-1}<x<c_{j}\right\} \neq \varnothing$. By strong $\mathcal{O}$-minimality, each element of $I_{j}$ must satisfy the same nonalgebraic principal type, and so, by Lemma 5.5(a), $I_{j}$ must be either dense or discrete without endpoints. However, were $I_{j}$ discrete, then the algebraic closure of any point in $I_{j}$ would be infinite. This, however, would violate Lemma 6.3, applying Ehrenfeucht's characterization of $\boldsymbol{\aleph}_{0}$-categoricity once again. So $I_{j}$ must be dense without endpoints, completing the proof of the lemma.

For the remainder of the section, we now fix in $\mathscr{M} C=\left\{c_{0}, \ldots, c_{n}\right\}$ and the $I_{j}$ 's, which we hence will call components, as guaranteed by 6.4 . Let the collection of nonempty $I_{j}$ 's be enumerated by $\left\{I_{j_{1}}, \ldots, I_{j_{p}}\right\}$.

LEMMA 6.5. For any $I_{j}$, and any $m<\omega$, every $m$-tuple of increasing elements in $I_{j}$ satisfies the same type.

Proof. For a contradiction, let $m \geqslant 1$ be such that every increasing $m$-tuple of elements in $I_{j}$ satisfies the same type, but the same is not true for increasing $m+1$-tuples of elements in $I_{j}$. Strong 0 -minimality then implies that for any $a_{1}, \ldots, a_{m} \in I_{j}, \quad\left(\operatorname{cl}\left(\left\{a_{1}, \ldots, a_{m}\right\}\right) \cap I_{j}\right) \backslash\left\{a_{1}, \ldots, a_{m}\right\} \neq \varnothing$. Let $a_{m+1} \in$ $\left(\operatorname{cl}\left(\left\{a_{1}, \ldots, a_{m}\right\}\right) \cap I_{j}\right) \backslash\left\{a_{1}, \ldots, a_{m}\right\}$. The uniqueness of the type of an increasing $m$-tuple from $I_{j}$ and the Exchange Principle, Theorem 4.1, together imply that we may assume $a_{1}<a_{2}<\cdots<a_{m+1}$ and that there is some definable (partial) function $f$ so that $f\left(a_{1}, \ldots, a_{m}\right)=a_{m+1}$. We then can define an increasing sequence of points $a_{m+j}=f\left(a_{j}, \ldots, a_{j+m-1}\right)$, for each $j \geqslant 1$, in $I_{j}$. Clearly, for each $j$, $a_{m+1} \in \operatorname{cl}\left(\left\{a_{1}, \ldots, a_{m}\right\}\right)$. However, this implies that $\operatorname{cl}\left(\left\{a_{1}, \ldots, a_{m}\right\}\right)$ is infinite, which cannot be, by Ehrenfeucht's characterization of $\boldsymbol{\aleph}_{0}$-categoricity, once again. Having reached a contradiction, the lemma is proved.

We shall say that a subset $\left\{I_{k_{1}}, \ldots, I_{k_{r}}\right\} \subseteq\left\{I_{j_{1}}, \ldots, I_{j_{p}}\right\}$ is weakly orthogonal if every $r$-tuple $\left(a_{1}, \ldots, a_{r}\right) \in I_{k_{1}} \times \cdots \times I_{k_{r}}$ satisfies the same type. Such a set $\left\{I_{k_{1}}, \ldots, I_{k_{r}}\right\}$ will be said to be orthogonal if for every sequence $\left(n_{1}, \ldots, n_{r}\right) \in \omega^{r}$, every properly ordered $\left(n_{1}+\cdots+n_{r}\right)$-tuple

$$
\left(a_{1}^{1}, a_{1}^{2}, \ldots, a_{1}^{n_{1}}, \ldots, a_{r}^{1}, a_{r}^{2}, \ldots, a_{r}^{n_{r}}\right) \in\left(I_{k_{1}}\right)^{n_{1}} \times \cdots \times\left(I_{k_{r}}\right)^{n_{r}}
$$

satisfies the same type. 
LEMMA 6.6. If $\left\{I_{k_{1}}, \ldots, I_{k_{r}}\right\}$ is weakly orthogonal, then it is orthogonal.

Proof. The proof proceeds by induction on $r \geqslant 1$. The case $r=1$ is a consequence of Lemma 6.5. Suppose, then, that the lemma has been established for sets of $r$ components, and we show that it is true for sets of $r+1$ components $\left\{I_{k_{1}}, \ldots, I_{k_{r+1}}\right\}$.

By weak orthogonality of $\left\{I_{k_{1}}, \ldots, I_{k_{r}}\right\}$, any $r$-tuple $\left(a_{1}, \ldots, a_{r}\right) \in I_{k_{1}} \times \cdots \times I_{k_{r}}$ satisfies the same type in $\mathscr{M}$. Furthermore, the weak orthogonality of $\left\{I_{k_{1}}, \ldots, I_{k_{r+1}}\right\}$ ensures that for any such $\left(a_{1}, \ldots, a_{r}\right), I_{k_{r+1}}$ remains a component in the $\boldsymbol{\aleph}_{0}$-categorical structure $\left(\mathscr{M}, a_{1}, \ldots, a_{r}\right)$ obtained by expanding $\mathscr{M}$ by constants for $a_{1}, \ldots, a_{r}$. Consequently, for any $\left(a_{1}, \ldots, a_{r}\right),\left(a_{1}^{\prime}, \ldots, a_{r}^{\prime}\right) \in I_{k_{1}} \times \cdots \times I_{k_{r}},\left(\mathscr{M}, a_{1}, \ldots, a_{r}\right)$ is isomorphic to $\left(\mathscr{M}, a_{1}^{\prime}, \ldots, a_{r}^{\prime}\right)$ via an isomorphism that maps $I_{k_{r+1}}$ onto itself. Also, by an application of Lemma 6.5 to $\left(\mathscr{M}, a_{1}, \ldots, a_{r}\right)$, it follows that for any $n_{r+1}<\omega$, every increasing $n_{r+1}$-tuple from $I_{k_{r+1}}$ satisfies the same type in $\left(\mathscr{M}, a_{1}, \ldots, a_{r}\right)$. Therefore, for any $n_{r+1}<\omega,\left(a_{r+1}^{1}, \ldots, a_{r+1}^{n_{r+1}}\right),\left(\left(a_{r+1}^{1}\right)^{\prime}, \ldots,\left(a_{r+1}^{n_{r+1}}\right)^{\prime}\right) \in\left(I_{k_{r+1}}\right)^{n_{r+1}}$, and $\left(a_{1}, \ldots, a_{r}\right),\left(a_{1}^{\prime}, \ldots, a_{r}^{\prime}\right) \in I_{k_{1}} \times \cdots \times I_{k_{r}}$, the $\left(r+n_{r+1}\right)$-tuples $\left(a_{1}, \ldots, a_{r}, a_{r+1}^{1}, \ldots, a_{r+1}^{n_{r+1}}\right)$ and $\left(a_{1}^{\prime}, \ldots, a_{r}^{\prime},\left(a_{r+1}^{1}\right)^{\prime}, \ldots,\left(a_{r+1}^{n_{r+1}}\right)^{\prime}\right)$ satisfy the same type in $\mathscr{M}$. In particular, observe for any $\left(a_{r+1}^{1}, \ldots, a_{r+1}^{n_{r+1}}\right) \in\left(I_{k_{r+1}}\right)^{n_{r+1}}$ that $\left\{I_{k_{1}}, \ldots, I_{k_{r}}\right\}$ remains a set of weakly orthogonal components in $\left(\mathscr{M}, a_{r+1}^{1}, \ldots, a_{r+1}^{n_{r+1}}\right)$ and, hence by induction hypothesis applied to $\left(\mathscr{M}, a_{r+1}^{1}, \ldots, a_{r+1}^{n_{r+1}}\right)$, that $\left\{I_{k_{1}}, \ldots, I_{k_{r}}\right\}$ is an orthogonal set of components in $\left(\mathscr{M}, a_{r+1}^{1}, \ldots, a_{r+1}^{n_{r+1}}\right)$. It then easily follows that $\left\{I_{k_{1}}, \ldots, I_{k_{r+1}}\right\}$ is an orthogonal set of components in $\mathscr{M}$, which we were to prove.

LEMMA 6.7. The set of components $\left\{I_{k_{1}}, I_{k_{2}}\right\}$ is weakly orthogonal if and only if there is no definable monotone bijection $f: I_{k_{1}} \rightarrow I_{k_{2}}$.

PROOF. If $\left\{I_{k_{1}}, I_{k_{2}}\right\}$ is weakly orthogonal, then for any $a \in I_{k_{1}}$, strong $\mathcal{O}$-minimality implies that $\operatorname{cl}(\{a\}) \cap I_{k_{2}}=\varnothing$, and surely there can be no such $f$. On the other hand, suppose that $\left\{I_{k_{1}}, I_{k_{2}}\right\}$ is not weakly orthogonal. Then, strong $\mathcal{O}$-minimality implies that $\operatorname{cl}(\{a\}) \cap I_{k_{2}} \neq \varnothing$, and an application of Lemma 5.5(b) produces the desired bijection.

LEMMA 6.8. Suppose that $I_{k_{1}}$ and $I_{k_{2}}$ are components in $\mathscr{M}$ and $f_{1}, f_{2}: I_{k_{1}} \rightarrow I_{k_{2}}$ are definable monotone bijections. Then $f_{1}=f_{2}$.

Proof. For a contradiction, suppose not. Then, for any $a \in I_{k_{1}}, f_{1}(a) \neq f_{2}(a)$. However, this implies that $f_{1}^{-1}\left(f_{2}(a)\right) \in \operatorname{cl}(\{a\}) \cap I_{k_{1}}$ is different from $a$, which is impossible by virtue of Lemma 6.5.

We now state and prove the most difficult lemma needed for the proof of Theorem 6.1.

Lemma 6.9. Let $I_{k_{1}}, \ldots, I_{k_{r}}$ be components of $\mathscr{M}$ such that for any $i, j \leqslant r, i \neq j$, $\left\{I_{k_{i}}, I_{k}\right\}$ are weakly orthogonal. Then $\left\{I_{k_{1}}, \ldots, I_{k_{r}}\right\}$ is an orthogonal set of components.

Proof. From Lemma 6.6, it follows that we need only prove that $\left\{I_{k_{1}}, \ldots, I_{k_{r}}\right\}$ is weakly orthogonal. This will be done by induction on $r \geqslant 2$. For $r=2$, this is just the hypothesis of the lemma. 
Assume, then, that the conclusion of the lemma has been established for sets of $r$ components, and we proceed to prove it for sets of $r+1$ components, $\left\{I_{k_{1}}, \ldots, I_{k_{r+1}}\right\}$. It clearly is enough to show that if $a_{1} \in I_{k_{1}}, \ldots, a_{r-2} \in I_{k_{r-2}}, b_{1} \in I_{k_{r-1}}, b_{2} \in I_{k_{r}}$, then $\operatorname{cl}\left(\left\{a_{1}, \ldots, a_{r-2}, b_{1}, b_{2}\right\}\right) \cap I_{k_{r+1}}=\varnothing$. For a contradiction, let us suppose that there are such $a_{1}, \ldots, a_{r-2}, b_{1}, b_{2}$, and some $b_{3} \in I_{k_{r+1}}$ so that $b_{3} \in$ $\operatorname{cl}\left(\left\{a_{1}, \ldots, a_{r-2}, b_{1}, b_{2}\right\}\right)$. For simplicity of notation, we work in the $\boldsymbol{\aleph}_{0}$-categorical structure $\mathscr{M}^{\prime}=\left(\mathscr{M}, a_{1}, \ldots, a_{r-2}\right)$. Induction hypothesis guarantees that each of $I_{k_{r-1}}, I_{k_{r}}$ and $I_{k_{r+1}}$ remains a component in $\mathscr{M}^{\prime}$, and furthermore, that any pair from $\left\{I_{k_{r},}, I_{k_{r}}, I_{k_{r+1}}\right\}$ is a weakly orthogonal pair in $\mathscr{M}^{\prime}$. Again for simplicity of notation for the remainder of the argument let us rename $I_{k_{r-1}}, I_{k_{r}}$ and $I_{k_{r+1}}$ to be $I_{1}, I_{2}$ and $I_{3}$, respectively.

Let $f$ be the definable (partial) function in $\mathscr{M}^{\prime}$ such that $f\left(b_{1}, b_{2}\right)=b_{3}$. By the weak orthogonality of $\left\{I_{1}, I_{2}\right\}, f$ must be a function from $I_{1} \times I_{2}$ to $I_{3}$. The weak orthogonality of both $\left\{I_{1}, I_{2}\right\}$ and $\left\{I_{1}, I_{3}\right\}$ implies that for any $a_{1} \in I_{1}$, both $I_{2}$ and $I_{3}$ remain components in $\left(\mathscr{M}^{\prime}, a_{1}\right)$. Therefore, by familiar arguments, for any $a_{1} \in I_{1}$, the function $f_{a_{1}}: I_{2} \rightarrow I_{3}$ given by $f_{a_{1}}\left(a_{2}\right)=f\left(a_{1}, a_{2}\right)$ is a monotone bijection. Since $I_{1}$ is a component in $\mathscr{M}^{\prime}$, it furthermore must be true that either for all $a_{1} \in I_{1}, f_{a_{1}}$ is monotonically increasing, or, for all $a_{1} \in I_{1}, f_{a_{1}}$ is monotonically decreasing. For the remainder of the argument, we suppose that the first alternative holds; if the second should hold, the argument would be similar. Observe that the same reasoning applies to the family of functions $f_{a_{2}}: I_{1} \rightarrow I_{3}$ for $a_{2} \in I_{2}$, given by $f_{a_{2}}\left(a_{1}\right)=f\left(a_{1}, a_{2}\right)$.

Now let us fix $c_{1} \in I_{1}, c_{2} \in I_{2}$ and $c_{3} \in I_{3}$ so that $f\left(c_{1}, c_{2}\right)=c_{3}$, and also let us choose some $c_{4} \neq c_{3}$ in $I_{3}$. Let $D=\operatorname{cl}\left(\left\{c_{1}, c_{2}, c_{3}, c_{4}\right\}\right)$ in $\mathscr{M}^{\prime}$. By the $\boldsymbol{N}_{0}$-categoricity of $\mathscr{M}^{\prime}, D$ must be finite, again by Ehrenfeucht's characterization. Obviously, for each $a_{1} \in D \cap I_{1}$ (respectively, $a_{2} \in D \cap I_{2}$ ), $f_{a_{1}}$ (respectively, $f_{a_{2}}$ ) is a bijection from $D \cap I_{2}$ (respectively, $D \cap I_{1}$ ) to $D \cap I_{3}$. In particular $\left|D \cap I_{1}\right|=\left|D \cap I_{2}\right|=$ $\left|D \cap I_{3}\right| \geqslant 2$. Let $a \in D \cap I_{1}$ and let $b \in D \cap I_{2}$ be the least element of $D \cap I_{2}$. Then, since it has been assumed that $f_{a}$ is monotonically increasing, we see that $c=f(a, b)=f_{a}(b)$ must be the least element of $D \cap I_{3}$. Now, let $a^{\prime} \neq a$ be an element of $D \cap I_{1}$. Since $f_{b}: I_{1} \rightarrow I_{3}$ is bijective, $f\left(a^{\prime}, b\right)=f_{b}\left(a^{\prime}\right)>c$. But then, since $f_{a^{\prime}}$ is monotonically increasing, and $b$ is the least element in $D \cap I_{2}$, it cannot be true that $f_{a^{\prime}}$ is a bijection from $D \cap I_{2}$ to $D \cap I_{3}$, a contradiction, completing the induction and the proof of the lemma.

We finally are able to complete the proof of Theorem 6.1.

Proof of 6.1. First, Lemma 6.4 established that (i) has been satisfied. Let

$$
E=\left\{(i, j): I_{i} \neq \varnothing \wedge I_{j} \neq \varnothing \wedge\left\{I_{i}, I_{j}\right\} \text { is not weakly orthogonal }\right\} .
$$

Lemma 6.7 ensures that $E$ is an equivalence relation, and Lemma 6.8 yields the uniqueness and the compositionality assertions about the bijections in (ii). It remains only to verify that $T$ admits the stated elimination of quantifiers. We prove that the complete type of any $p$-tuple, $\left(a_{1}, \ldots, a_{p}\right)$, of elements of $\mathscr{M}$ is implied by the formula $\Psi$ consisting of the conjunction of all instances and negated instances of formulas of the form $x=y, x<y, \underline{c}_{i}<x, x<\underline{c}_{i}, y=\underline{f}_{i, j}(x), y<\underline{f}_{i, j}(x)$ and $\underline{f}_{i, j}(x)<y$, that hold of the coordinates of $\left(a_{1}, \ldots, a_{p}\right)$. 
To prove this, let us rewrite $\left(a_{1}, \ldots, a_{p}\right)$ as $\left(a_{1}^{\prime}, \ldots, a_{1}^{n_{1}}, \ldots ; a_{r}^{1}, \ldots, a_{r}^{n_{r}}\right)$ such that for every $j=1, \ldots, r$ the components of $a_{j}^{1}, \ldots, a_{j}^{n_{j}}$ all lie in the same $E$-equivalence class (we may neglect any members in $\left(a_{1}, \ldots, a_{p}\right)$ that are in $\left\{c_{1}, \ldots, c_{n}\right\}$, of course). First, we claim that for any $j=1, \ldots, r$, if $\psi_{j}$ consists of those conjuncts of $\Psi$ involving only $a_{j}^{1}, \ldots, a_{j}^{n_{j}}$, then $\psi_{j}$ implies the complete type of $\left(a_{j}^{1}, \ldots, a_{j}^{n_{j}}\right)$. This follows easily as a consequence of Lemma 6.5 , because if it were not true, then by appropriate use of the $f_{i, j}$ 's, there would exist two increasing $n_{j}$-tuples of elements of some component that satisfied different types. Secondly, the existence of two $p$-tuples $\left(a_{1}^{\prime}, \ldots, a_{r}^{n_{r}}\right)$ and $\left(b_{1}^{\prime}, \ldots, b_{r}^{n_{r}}\right)$ both satisfying $\Psi$ but yet having different types would imply, by the use of the $f_{i, j}$ 's once again, the existence of two such $p$-tuples so that for every $j=1, \ldots, r$ all coordinates of $\left(a_{j}^{1}, \ldots, a_{j}^{n_{j}}\right)$ and $\left(b_{j}^{1}, \ldots, b_{j}^{n_{j}}\right)$ lie in the same component in addition to satisfying the same type. However, this cannot be possible according to Lemma 6.9 .

\section{REFERENCES}

0. J. Baldwin, Stability theory, Perspectives in Mathematical Logic, Springer-Verlag, Berlin (to appear).

1. J. Baldwin and A. Lachlan, On strongly minimal sets, J. Symbolic Logic 36 (1971), 79-96.

2. P. Cohn, Algebra, Vol. 2, Wiley, London, 1977.

3. L. van den Dries, Remarks on Tarski's problem concerning $(R,+, \cdot, \exp )$, manuscript, 1983.

4. P. Erdös, L. Gillman and M. Henriksen, An isomorphism theorem for real closed fields, Ann. of Math. (2) 61 (1955), 542-554.

5. S. Feferman and R. Vaught, The first order properties of algebraic systems, Fund. Math. 47 (1959), 57-103.

6. F. Gausdorff, Grundzuge der Mengenlehre, Leipzig, 1914.

7. C. Langford, Some theorems on deducibility, Ann. of Math. (2) 28 (1927), 16-40.

8. A. Macintyre, On $\aleph_{1}$-categorical theories of fields, Fund. Math. 71 (1971), 1-25.

9. A. Pillay, An introduction to stability theory, Oxford Univ. Press, Oxford, 1983.

10. A. Pillay and C. Steinhorn, Definable sets in ordered structures, Bull. Amer. Math. Soc. (N.S.) 11 (1984).

11. K-P. Podewski, Minimale ringe, preprint 1973.

12. M. Rabin, Decidable theories, Handbook of Mathematical Logic (J. Barwise ed.), North-Hollar,d, Amsterdam, 1977.

13. J. Reineke, Minimale gruppen, Z. Math. Logik Grundlag. Math. 21 (1975), 357-359.

14. A. Robinson, Complete theories, North-Holland, Amsterdam, 1956.

15. A. Tarski and J. McKinsey, A decision problem for elementary algebra and geometry, Rand Corporation, Santa Monica, 1948.

16. S. Shelah, Uniqueness and characterization of prime models over sets for totally transcendental first-order theories, J. Symbolic Logic 37 (1972), 107-113.

17. G. Sacks, Saturated model theory, Benjamin, Reading, Mass., 1972.

Department of Mathematics, University of Notre Dame, Notre Dame, Indiana 46556

Department of Mathematics, Vassar College, Poughkeepsie, New York 12601 